\title{
Bayesian selection of multiresponse nonlinear regression model
}

\author{
Vivien Rossi ${ }^{\mathrm{a}}$ and Jean-Pierre Vila ${ }^{\mathrm{b} *}$ \\ ${ }^{a}$ UR Dynamique des forêts naturelles, CIRAD, Campus International de Baillarguet, 34398 Montpellier \\ cedex 5, France; ${ }^{b}$ UMR Analyse des Systèmes et de Biométrie, INRA-ENSAM, 2 Place P. Viala, 34060 \\ Montpellier cedex 1, France
}

(Received 14 August 2006; final version received 22 August 2007)

\begin{abstract}
A Bayesian method for the selection and ranking of multiresponse nonlinear regression models in a given model set is proposed. It uses an expected utility criterion based on the logarithmic score of a posterior predictive density of each model. Two approaches are proposed to get this posterior. The first is based on a general asymptotically convergent approximation of the model parameter posterior corresponding to a wide class of parameter priors. The second, a numerical one, uses well-known pure or hybrid MCMC methods. Both posterior approximation approaches allow the practical computation of the expected utility criterion on a sample-reuse basis. This leads to a class of Bayesian cross-validation (CV) procedures, aiming at finding the model having the best predictive ability among a set of models. Varied comparisons of the performances of the Bayesian procedures, with that of AIC, BIC and standard CV procedures, are proposed on a simulated and a real model selection problem case studies, of low and high parametric dimensions respectively.
\end{abstract}

Keywords: multiresponse nonlinear regression; Bayesian model selection; expected utility criterion; MCMC methods

\section{Introduction}

When it can be reduced to parameter hypothesis testing, nonlinear model selection can be performed through extensions of well-known asymptotic inferential tests as likelihood ratio, Wald's or Rao's Lagrange multiplier tests [1]. When selection has to be done among nonnested models other tools have to be used. Information-theoretic criteria as Akaike's and its various derivatives are then frequently used and offer easy-to-use procedures for selection of parsimonious models [2]. These criteria combine some measure of fit with a penalty term to account for model complexity. It is well known that depending on the kind of penalty used, each of these criteria performs well only in one type of situation. To counter this limitation, [3] proposed recently an adaptive selection procedure which combines the benefit of several of such criteria as Akaike's information criterion (AIC), Bayesian information criterion (BIC), Mallow's $C_{p}$ and risk inflation criterion (RIC). Many adaptive Bayesian variants of the previous penalized

*Corresponding author. Email: vila@supagro.inra.fr

ISSN 0233-1888 print/ISSN 1029-4910 online

(C) 2008 Taylor \& Francis

DOI: $10.1080 / 02331880701739824$

http://www.informaworld.com 
measure-of-fit criteria have also been considered, especially for variable selection in regression see [4-7] and the references therein. These last approaches, and recent ones based on bootstrapping and hypothesis testing [8], take advantage of the theoretical advances in resampling and Markov chain Monte Carlo methods. Most of these approaches have been developed for single-response models but their extension to the multiresponse case is generally straightforward. When the selection problem does not reduce to variable selection in multiple linear regression or comparison of nested models, the most favoured computer intensive procedure is cross validation (CV) [9], based on model prediction capability. Its reduced probability assumption requirements (as exchangeability assumption) makes classic CV particulary attractive. But $\mathrm{CV}$ is known to be inconsistent [10] as are all the methods asymptotically equivalent to it (e.g. AIC, $C_{p}$, the jackknife and the bootstrap). Moreover, $\mathrm{CV}$ is often too conservative (selection of unnecessary large models). Recourse to Bayesian variants of CV can help to reduce this loss of efficiency [9, 11, 12]. Furthermore in addition to point-prediction-based comparisons according to quadratic loss, the Bayesian approach can offer alternatives as comparisons resting upon predictive distributions, according to logarithmic or quadratic scores (see e.g. [13]). The greater richness of information attached to predictive distributions makes this last type of approach particularly attractive for model selection. Conjugate-prior-based Bayesian approaches of this type, have for example, been proposed for the comparison of feedforward neural network architectures $[14,15]$.

This paper is devoted to the study of generalizations of such Bayesian approaches for the selection and ranking of multiresponse nonlinear regression models. It uses a sample-reuse calculation of an expected utility built from the logarithmic score of a posterior predictive density, for each competing model. We consider two quite different approaches to get this posterior. The first is based on a general asymptotically convergent approximation of the model parameter posterior. This posterior parameter approximation itself is valid for a wide class of parameter priors. By so doing the critical issue of the choice of an appropriate prior for the model parameters and that of the calculation of the resulting posterior, are conveniently defused. The second approach to reach the posterior of interest, is a numerical one and uses well-known pure or hybrid MCMC methods from noninformative priors. More precisely, a mixed procedure combining the respective advantages of the Gibbs sampling and of the Metropolis-Hastings algorithm is considered, in addition to the standard Metropolis-Hastings algorithm.

The paper is organized as follows. In Section 2 the statistical framework of the multiresponse nonlinear regression within which the model selection problem is considered, is set up. In Section 3 the building elements of the expected-utility-based criterion are considered. The issue of the posterior predictive density to be used in the computation of the utility criterion is then examined in the next two sections. More precisely, in Section 4 a general convergent analytic approximation of a model parameter posterior which is valid for any usual prior is developed. A convergent analytic approximation of the related posterior predictive density is then built up under the form of a multivariate Student distribution. In Section 5, two numerical counterparts to this analytic approximation are considered through the MCMC procedures mentioned above. In Section 6 the three variants of our Bayesian selection procedure are applied to a simulated problem of multi-response regression model selection and then to an actual selection problem of a multioutput feedforward predictive neural network in a Soil Science study. These two case studies are representative of low and high parametric dimension situations respectively. It is shown how the performances of the Bayesian procedure compare advantageously with that of AIC, BIC and classic $\mathrm{CV}$ procedures on the same problems.

Appendix A provides the proofs of all lemmas, propositions and convergence theorems of expected utility criteria, parameter posterior and predictive density approximations.

Finally, Appendix B presents the results of the comparisons of all the model selection criteria considered, on the two case studies. 


\section{Multiresponse modeling framework}

We are interested in multiresponse nonlinear regression models of the form

$$
\text { Model } M: \quad y_{i}=f\left(x_{i}, \theta\right)+\varepsilon_{i}
$$

where for $i \in\{1, \ldots, n\}$

$$
y_{i} \in \mathbb{R}^{d} \quad x_{i} \in \mathbb{R}^{l} \quad \theta \in \Theta \subset \mathbb{R}^{s} \quad \varepsilon_{i} \sim N_{d}(0, \Sigma) \quad \Sigma \in \mathcal{S} \subset \mathbb{R}^{d \times d}
$$

where $\mathcal{S}$ is the set of all positive definite symmetric matrices of dimension $d \times d$. In this paper, we shall have to consider more often than the variance-covariance matrix $\Sigma$, the precision matrix $\Lambda=\Sigma^{-1}$.

Let us denote

- $Z_{n}=\left(x_{i}, y_{i}\right), i=1, \ldots, n$, the data set, made of $n$ i.i.d. random data points $(x, y)$.

- $\dot{f}_{x_{i}, \theta}=\left(\partial f\left(x_{i}, \theta\right) / \partial \theta_{j}\right) j \in\{1, \ldots, q\}$ when these derivatives exist.

Other notations:

- $\mathcal{N}_{s}(\cdot \mid \mu, \Sigma)$ : the $s$-dimensional Gaussian distribution with expectation $\mu$ and covariance matrix $\Sigma$.

- $\mathcal{W} i_{d}(\cdot \mid \alpha, \beta)$ : the $d$-dimensional Wishart distribution with parameters $\alpha$ and $\beta$.

- $S t_{d}(\cdot \mid \mu, \Psi, \alpha)$ : the $d$-dimensional Student distribution with parameters $\mu, \Psi$ and $\alpha$.

[1] show, how given $Z_{n}$, under model $M$ and under appropriate regularity conditions, consistent maximum likelihood estimates (or equivalent least squares estimates) $\hat{\theta}_{n}$ and $\widehat{\Lambda}_{n}$ of $\theta$ and $\Lambda$ respectively can be calculated, such that

$$
\begin{aligned}
\hat{\theta}_{n} & =\operatorname{argmin}_{\theta} \operatorname{det}\left[\sum_{i=1}^{n}\left(y_{i}-f\left(x_{i}, \theta\right)\right)\left(y_{i}-f\left(x_{i}, \theta\right)\right)^{\prime}\right] \\
\widehat{\Lambda}_{n}^{-1} & =\hat{\Sigma}_{n}=\frac{1}{n} \sum_{i=1}^{n}\left(y_{i}-f\left(x_{i}, \hat{\theta}_{n}\right)\right)\left(y_{i}-f\left(x_{i}, \hat{\theta}_{n}\right)\right)^{\prime}
\end{aligned}
$$

More generally when model $M$ is incorrect, there still exist values $\theta_{o}$ and $\Lambda_{o}$ to which the maximum likelihood estimates $\hat{\theta}_{n}$ and $\hat{\Lambda}_{n}$ converge almost surely with $n[16,17]$ : $\theta_{o}$ and $\Lambda_{o}$ are the parameter values minimizing the Kullback-Leibler information criterion between the true $(x, y)$ data distribution and the $(x, y)$ distribution induced by model $M$.

Given $Z_{n}$ and a set $\mathcal{M}$ of $J$ models $\left\{M^{j}, j=1, \ldots, J\right\}$, with $E\left(y \mid M^{j}, x\right)=f^{j}\left(x, \theta^{j}\right)$, the question of interest is to select the best model in some predictive sense.

\section{The expected-utility-based criterion}

To do this selection we follow the maximum-expected-utility approach [13] for which the optimal model choice is $M^{*}$ such that

$$
U\left(M^{*} \mid Z_{n}\right)=\sup _{M^{j} \in \mathcal{M}} U\left(M^{j} \mid Z_{n}\right)
$$

where

$$
U\left(M^{j} \mid Z_{n}\right)=\int u\left(M^{j}, y, x \mid Z_{n}\right) p\left((x, y) \mid Z_{n}\right) \mathrm{d} y \mathrm{~d} x
$$

with $u\left(M^{j}, y, x \mid Z_{n}\right)$ a given utility function and $p\left((x, y) \mid Z_{n}\right)$ a probability density representing actual beliefs about $(x, y)$ having observed $Z_{n}$. 
As we are interested in comparing models from a predictive distribution point of view we take as utility function the logarithmic score

$$
u\left(M^{j}, y, x \mid Z_{n}\right)=\log p\left(y \mid M^{j}, x, Z_{n}\right)=\log p_{j}\left(y \mid x, Z_{n}\right)
$$

where $\log p_{j}\left(y \mid x, Z_{n}\right)$ is the posterior predictive density of a response $y$ of model $M^{j}$ at $x$, given the past observations $Z_{n}$, a prior density $p(\theta, \Lambda)$ for the model $M^{j}$ parameters and its related posterior $p\left(\theta, \Lambda \mid Z_{n}\right)$.

With this choice for the utility function

$$
U_{n}^{j}=U\left(M^{j} \mid Z_{n}\right)=\int u\left(M^{j}, y, x \mid Z_{n}\right) p\left((x, y) \mid Z_{n}\right) \mathrm{d} y \mathrm{~d} x=E_{x, y}\left[\log p_{j}\left(y \mid x, Z_{n}\right)\right]
$$

Using this criterion implies that it can be computed for every $n$ and every possible data set $Z_{n}$. But $p\left((x, y) \mid Z_{n}\right)$ in Equation (6) is not available. We can only search for an estimate of $U_{n}^{j}$ for each $M^{j} \in \mathcal{M}$. To do this, we consider a well-known approximation through $\mathrm{CV}$.

\subsection{Expected utility approximation through $C V$}

$\mathrm{CV}$ predictive density methods for model comparison have been considered by several authors (see reviews of $[18,19])$.

Let us consider the $n$ partitions of $Z_{n}: Z_{n}=\left[Z_{n-1}[i],\left(x_{i}, y_{i}\right)\right]$ for $1 \leq i \leq n$, where $Z_{n-1}[i]$ denotes the data set $Z_{n}$ after withdrawal of the data point $\left(x_{i}, y_{i}\right)$. Let us denote $u_{i, n}^{j}=$ $\log p_{j}\left(y_{i} \mid x_{i}, Z_{n-1}[i]\right)$. The $\left\{u_{i, n}^{j}, i=1, \ldots, n\right\}$ constitute a collection of leave-one-out $\mathrm{CV}$ predictive densities. Let

$$
\hat{U}_{n}^{j}=\frac{1}{n} \sum_{i=1}^{n} u_{i, n}^{j}
$$

[13] proposed to use $\hat{U}_{n}^{j}$ as a good approximation of $U_{n}^{j}$ the expected utility of model $M^{j}$. More recently, [20,21] confirmed this recommendation and estimated its probability distribution.

By looking for the model $M^{j}$ which maximizes (7), the procedure selects, on a sample-reuse basis, the model under which the data set $Z_{n}$ achieves the highest level of some internal consistency: the best model is that which on the whole, most favours the likelihood of each observation with respect to the others.

The criterion (7) is based on the posterior predictive density $p_{j}\left(y \mid x, Z_{n}\right)$, where

$$
p_{j}\left(y \mid x, Z_{n}\right)=\int p_{j}(y \mid x, \theta, \Lambda) p_{j}\left(\theta, \Lambda \mid Z_{n}\right) \mathrm{d} \theta \mathrm{d} \Lambda
$$

in which $p_{j}\left(\theta, \Lambda \mid Z_{n}\right)$ is the parameter posterior induced by a given parameter prior $p_{j}(\theta, \Lambda)$. However, the choice of a relevant prior and the exact calculation of its posterior can be difficult, if not untractable. On these bases, the two next sections are devoted to convergent analytic and numerical approximations respectively, of $p_{j}\left(y \mid x, Z_{n}\right)$.

\section{4. $L_{1}$-convergent posterior predictive density approximations}

The first step of our approach is to show how a general $L_{1}$-convergent approximation of the parameter posterior density which is valid for a wide class of parameter priors, can be built up for each of the $J$ competing models $\left\{M^{j}, j=1, \ldots, J\right\}$ (the index $j$ will be dropped to alleviate notations in all the following). 


\subsection{A $L_{1}$-convergent approximation of the parameter posterior density}

Let $\mathcal{H}$ be the following set of assumptions for model $M^{j}$ :

$H_{1} \quad x_{i} \in \mathcal{X}$ a compact subset of $\mathbb{R}^{l}, i=1, \ldots, n$.

$H_{2}$ There exist consistent estimators of $\theta_{o}$ and $\Lambda_{o}$, model $M^{j}$ parameter values such as defined in Section 2. When $M^{j}$ is the true model, $\theta_{o}$ and $\Lambda_{o}$ are its parameter values.

$H_{3}$ The model function $f_{j}(x, \theta)$ is of class $C^{1}$ both in $x$ and $\theta$.

$H_{4}$ There exists a compact subset $C \subset \Theta \times \mathcal{S}$ including an open neighbourhood of $\left(\theta_{o}, \Lambda_{o}\right)$, such that $p(\theta, \Lambda)$ is bounded and strictly positive on $C$.

Let $p(\theta, \Lambda)$ be a given parameter prior. Now, by definition of a parameter posterior density for model (1)

$$
p\left(\theta, \Lambda \mid Z_{n}\right)=K_{n}|\Lambda|^{n / 2} \exp \left\{-\frac{1}{2} \sum_{i=1}^{n}\left\|y_{i}-f\left(x_{i}, \theta\right)\right\|_{\Lambda}^{2}\right\} p(\theta, \Lambda)
$$

where $K_{n}$ is a normalizing constant.

We consider an easily tractable approximate:

$$
\text { let } \begin{aligned}
\hat{p}\left(\theta, \Lambda \mid Z_{n}\right)= & \mathcal{N}_{s}\left(\theta \mid \hat{\theta}_{n},\left(\sum_{i=1}^{n} \dot{f}_{x_{i}, \hat{\theta}_{n}}^{\prime} \hat{\Lambda}_{n} \dot{f}_{x_{i}, \hat{\theta}_{n}}\right)^{-1}\right) \\
& \times \mathcal{W}_{i_{d}}\left(\Lambda \mid \frac{n+d+1}{2}, \frac{1}{2} \sum_{i=1}^{n}\left(y_{i}-f\left(x_{i}, \hat{\theta}_{n}\right)\right)\left(y_{i}-f\left(x_{i}, \hat{\theta}_{n}\right)\right)^{\prime}\right)
\end{aligned}
$$

Let $\hat{P}($.) be the related probability measure over $\mathcal{F}$ the sigma-algebra associated with $\Theta \times \mathcal{S}$. The following lemma ensures the consistency of $\hat{p}\left(\theta, \Lambda \mid Z_{n}\right)$ to $\theta_{o}, \Lambda_{o}$.

Lemma 4.1 Suppose assumptions $\mathcal{H}$ are satisfied. Let $A$ be any measurable subset of $\Theta \times \mathcal{S}$ including an open neighbourhood of $\left(\theta_{o}, \Lambda_{o}\right)$. Then $\lim _{n \rightarrow \infty} \hat{P}(A)=1$ a.s.

To ensure that $\hat{p}\left(\theta, \Lambda \mid Z_{n}\right)$ is a consistent estimate of $p\left(\theta, \Lambda \mid Z_{n}\right)$, let us consider the following prior-free approximation of $p\left(\theta, \Lambda \mid Z_{n}\right)$

$$
\tilde{p}\left(\theta, \Lambda \mid Z_{n}\right)=\tilde{K}_{n}|\Lambda|^{n / 2} \exp \left\{-\frac{1}{2} \sum_{i=1}^{n}\left\|y_{i}-f\left(x_{i}, \theta\right)\right\|_{\Lambda}^{2}\right\} \times I_{C_{\mathcal{S}}}(\theta, \Lambda)
$$

where $\tilde{K}_{n}$ is a normalizing constant and $I_{C_{\mathcal{S}}}$ is the indicator function of $C_{\mathcal{S}}$, with $C_{S}=\operatorname{Proj}_{\Theta}(C) \times$ $\mathcal{S}$, where $\operatorname{Proj}_{\Theta}(C)$ denotes the projection of $C$ upon $\Theta$.

With respect to Equation (9) that amounts to take an improper prior with density equal to one on $C_{\mathcal{S}}$ and zero elsewhere.

This prior-free approximation (11) of $p\left(\theta, \Lambda \mid Z_{n}\right)$ allows to characterize the asymptotic behaviour of the $L_{1}$ distance over $C$, between $\hat{p}\left(\theta, \Lambda \mid Z_{n}\right)$ and $p\left(\theta, \Lambda \mid Z_{n}\right)$ as the number of data $n$ grows to infinity. This is done through the following three propositions.

Proposition 4.2 Under assumptions $\mathcal{H}$

$$
\lim _{n \rightarrow \infty} \int_{C}\left|\tilde{p}\left(\theta, \Lambda \mid Z_{n}\right)-p\left(\theta, \Lambda \mid Z_{n}\right)\right| \mathrm{d} \theta \mathrm{d} \Lambda=0 \text { a.s. }
$$


PROPOSITION 4.3 Under assumptions $\mathcal{H}$

$$
\lim _{n \rightarrow \infty} \int_{C}\left|\hat{p}\left(\theta, \Lambda \mid Z_{n}\right)-\tilde{p}\left(\theta, \Lambda \mid Z_{n}\right)\right| \mathrm{d} \theta \mathrm{d} \Lambda=0 \text { a.s. }
$$

PROPOSITION 4.4 Under assumptions $\mathcal{H}$

$$
\lim _{n \rightarrow \infty} \int_{C}\left|\hat{p}\left(\theta, \Lambda \mid Z_{n}\right)-p\left(\theta, \Lambda \mid Z_{n}\right)\right| \mathrm{d} \theta \mathrm{d} \Lambda=0 \text { a.s. }
$$

Proposition 4.4 is an immediate consequence of Proposition 4.2 and Proposition 4.3, by noting that

$$
|\hat{p}-p| \leq|\hat{p}-\tilde{p}|+|\tilde{p}-p|
$$

Proposition 4.4 makes it possible to get the $L_{1}$ consistency of $\hat{p}\left(\theta, \Lambda \mid Z_{n}\right)$ as $n$ grows to infinity:

THEOREM 4.5 Under assumptions $\mathcal{H}$

$$
\lim _{n \rightarrow \infty} \int\left|\hat{p}\left(\theta, \Lambda \mid Z_{n}\right)-p\left(\theta, \Lambda \mid Z_{n}\right)\right| \mathrm{d} \theta \mathrm{d} \Lambda=0 \quad \text { a.s. }
$$

Proof Let $C^{c}$ be the subset of $\Theta \times \mathcal{S}$ complementary to $C$.

$$
\begin{aligned}
\int\left|\hat{p}\left(\theta, \Lambda \mid Z_{n}\right)-p\left(\theta, \Lambda \mid Z_{n}\right)\right| \mathrm{d} \theta \mathrm{d} \Lambda & =\int_{C}|\hat{p}-p|+\int_{C^{c}}|\hat{p}-p| \\
& \leq \int_{C}|\hat{p}-p|+\int_{C^{c}} \hat{p}+\int_{C^{c}} p
\end{aligned}
$$

By Proposition 4.4 the first integral in Equation (15) tends to zero as $n$ tends to $\infty$. By Lemma 4.1 the second integral in Equation (15) tends to zero as $n$ tends to $\infty$ since $\left(\theta_{o}, \Lambda_{o}\right)$ does not belong to $C^{c}$. The same is true for the third integral in Equation (15) since $p$ is consistent $[17,22,23]$ and $\left(\theta_{o}, \Lambda_{o}\right)$ does not belong to $C^{c}$.

\subsection{Application to $L_{1}$-approximations of the posterior predictive density}

Thanks to the consistent estimate of $p\left(\theta, \Lambda \mid Z_{n}\right)$ provided in the previous section, it is now possible to get a consistent estimate of the density of interest $p\left(y \mid x, Z_{n}\right)$.

By definition

$$
p\left(y \mid x, Z_{n}\right)=\int p(y \mid x, \theta, \Lambda) p\left(\theta, \Lambda \mid Z_{n}\right) \mathrm{d} \theta \mathrm{d} \Lambda
$$

THEOREM 4.6 Let $\hat{p}\left(\theta, \Lambda \mid Z_{n}\right)$ be a general $L_{1}$-convergent approximation of the parameter posterior density $p\left(\theta, \Lambda \mid Z_{n}\right)$ and let

$$
\hat{p}\left(y \mid x, Z_{n}\right)=\int \hat{p}(y \mid x, \theta, \Lambda) \hat{p}\left(\theta, \Lambda \mid Z_{n}\right) \mathrm{d} \theta \mathrm{d} \Lambda
$$

with

$$
\hat{p}(y \mid x, \theta, \Lambda)=\frac{|\Lambda|^{1 / 2}}{(2 \pi)^{d / 2}} \exp \left\{-\frac{1}{2}\left\|y-f\left(x, \hat{\theta}_{n}\right)\right\|_{\Lambda}^{2}\right\} .
$$

Then

$$
\lim _{n \rightarrow \infty} \int\left|\hat{p}\left(y \mid x, Z_{n}\right)-p\left(y \mid x, Z_{n}\right)\right| \mathrm{d} y=0 \quad \text { a.s. }
$$


With the help of both Theorem 4.5 and Theorem 4.6 it is possible to get a more practical $L_{1}$-convergent approximation of the posterior predictive density under the form of a multivariateStudent distribution.

COROLlary 4.7 Under assumptions $\mathcal{H}$, let

$$
\hat{p}_{n}\left(y \mid x, Z_{n}\right)=S t_{d}\left(y \mid f\left(x, \hat{\theta}_{n}\right), \frac{n+2}{n} \hat{\Lambda}_{n}, n+2\right)
$$

then

$$
\lim _{n \rightarrow \infty} \int\left|\hat{p}_{n}\left(y \mid x, Z_{n}\right)-p\left(y \mid x, Z_{n}\right)\right| \mathrm{d} y=0 \quad \text { a.s. }
$$

Proof Bringing $\hat{p}\left(\theta, \Lambda \mid Z_{n}\right)$ as given by Equation (10) into (17), easily leads to $\hat{p}_{n}\left(y \mid x, Z_{n}\right)$ as given by Equation (20).

\section{Numerical posterior predictive density approximations by an MCMC approach}

Under the assumption $\varepsilon_{i} \sim N_{d}(0, \Sigma)$ as in Equation (1), it is possible to use an MCMC method to approximate the posterior predictive density. Remember that

$$
p\left(y \mid x, Z_{n}\right)=\int p(y \mid x, \theta, \Lambda) p\left(\theta, \Lambda \mid Z_{n}\right) \mathrm{d} \theta \mathrm{d} \Lambda
$$

Let $\left\{\left(\theta^{1}, \Lambda^{1}\right), \ldots,\left(\theta^{m}, \Lambda^{m}\right)\right\}$ be a sample from $p\left(\theta, \Lambda \mid Z_{n-1}[i]\right)$. By the strong law of large numbers we have

$$
\lim _{m \rightarrow \infty} \frac{1}{m} \sum_{k=1}^{m} p\left(y_{i} \mid x_{i}, \theta^{k}, \Lambda^{k}\right)=p\left(y_{i} \mid x_{i}, Z_{n-1}[i]\right) \quad \text { a.s. }
$$

The density $p\left(\theta, \Lambda \mid Z_{n}\right)$ as in Equation (9), is only known up to a normalizing constant, and cannot directly provide a sample $\left\{\theta^{k}, \Lambda^{k}\right\}$. However, one can get an approximately $p\left(\theta, \Lambda \mid Z_{n-1}[i]\right)$ distributed sample thanks to an MCMC method as the Metropolis-Hastings algorithm [24] or, as will be shown, a hybrid Gibbs-Hastings algorithm [25, 26] under certain conditions for the parameter prior $p(\theta, \Lambda)$. Nevertheless, such MCMC integrations can suffer from instability [19] and heavy computing. The interpretation of the utility criterion approximated by such MCMC methods can thus be delicate. However, in order to compare these now well-known approaches with the analytic ones developed previously, we implemented them, when allowed by the application considered (see Section 6).

\subsection{Metropolis-Hastings approximate sampling from $p\left(\theta, \Lambda \mid Z_{n}\right)$}

First, let us introduce briefly the principle of the Metropolis-Hastings algorithm in our context

(i) $(\tilde{\theta}, \tilde{\Lambda}) \sim q\left(\theta, \Lambda \mid \theta^{t}, \Lambda^{t}\right)$

(ii) $\left(\theta^{t+1}, \Lambda^{t+1}\right)= \begin{cases}\left(\theta^{t}, \Lambda^{t}\right) & \text { with probability } 1-\rho \\ (\tilde{\theta}, \tilde{\Lambda}) & \text { with probability } \rho\end{cases}$ 
with

$$
\rho=1 \wedge \frac{p\left(\tilde{\theta}, \tilde{\Lambda} \mid Z_{n}\right)}{p\left(\theta^{t}, \Lambda^{t} \mid Z_{n}\right)} \frac{q\left(\theta^{t}, \Lambda^{t} \mid \tilde{\theta}, \tilde{\Lambda}\right)}{q\left(\tilde{\theta}, \tilde{\Lambda} \mid \theta^{t}, \Lambda^{t}\right)}
$$

The density $q\left(\theta, \Lambda \mid \theta^{t}, \Lambda^{t}\right)$ is generally called the proposal distribution. The Markov chain $\left\{\left(\theta^{t}, \Lambda^{t}\right), t \in \mathbb{N}\right\}$ thus generated, admits $p\left(\theta, \Lambda \mid Z_{n}\right)$ as stationary distribution if the support of $q$ contains the support of $p\left(\theta, \Lambda \mid Z_{n}\right)$. See [25] or [26] for more details and for the theoretical study of this algorithm.

In our context, the density $p\left(\theta, \Lambda \mid Z_{n}\right)$ is only known up to a normalizing constant

$$
\begin{aligned}
p\left(\theta, \Lambda \mid Z_{n}\right) & =\frac{p\left(Z_{n} \mid \theta, \Lambda\right) p(\theta, \Lambda)}{\int p\left(Z_{n} \mid \theta, \Lambda\right) p(\theta, \Lambda) \mathrm{d} \theta \mathrm{d} \Lambda} \\
& \propto p\left(Z_{n} \mid \theta, \Lambda\right) p(\theta, \Lambda)
\end{aligned}
$$

which does not impede the use of the Metropolis-Hastings algorithm, since only the ratio $p\left(\tilde{\theta}, \tilde{\Lambda} \mid Z_{n}\right) / p\left(\theta^{t}, \Lambda^{t} \mid Z_{n}\right)$ is considered, in Equation (22).

For computational efficiency, the distribution $q$ should be chosen so that it can be easily evaluated and sampled. As the convergence is faster when $q$ is close to $p\left(\theta, \Lambda \mid Z_{n}\right)$ (see [26]), a good candidate for the proposal distribution in our case is the following

$$
q\left(\theta^{t+1}, \Lambda^{t+1} \mid \theta^{t}, \Lambda^{t}\right)=\mathcal{N}_{s}\left(\theta^{t+1} \mid \theta^{t}, V^{t}\right) \mathcal{W} i_{d}\left(\Lambda^{t+1} \mid \frac{n+d+1}{2}, \frac{n}{2}\left(\Lambda^{t}\right)^{-1}\right)
$$

with $V^{t}=\left(\sum_{i=1}^{n} \dot{f}_{x_{i}, \theta^{t}}^{\prime} \Lambda^{t} \dot{f}_{x_{i}, \theta^{t}}\right)^{-1}$. Such a proposal distribution is close to $\hat{p}\left(\theta, \Lambda \mid Z_{n}\right)$ in Equation (10), which is itself a convergent approximation of $p\left(\theta, \Lambda \mid Z_{n}\right)$.

The choice of the prior distribution, $p(\theta, \Lambda)$ is delicate. For instance Jeffrey's priors, which are invariant to parameter transformation, are not adapted to our context. First, Jeffrey's principle is controversial for multiparameter models [27]. Second, Jeffrey's noninformative prior density, $p(\theta, \Lambda) \propto|J(\theta, \Lambda)|^{1 / 2}$, where $J(\theta, \Lambda)$ is the Fisher information matrix, is most often untractable because of the general nonlinearity with respect to $\theta$. It reduces to $p(\theta, \Lambda) \propto|\Lambda|^{(d+1) / 2}$ when $\theta$ is discarded but it has nevertheless to be proscribed since by (23) $p(\theta, \Lambda)$ must be such that $\int p\left(Z_{n} \mid \theta, \Lambda\right) p(\theta, \Lambda) \mathrm{d} \theta \mathrm{d} \Lambda<\infty$.

A prior which preferentially weights a theoretical neighbourhood of the true parameter values is of particular interest. A good candidate is

$$
\begin{aligned}
p(\theta, \Lambda)= & \mathcal{N}_{s}\left(\theta \mid \hat{\theta}_{n},\left(\sum_{i=1}^{n} \dot{f}_{x_{i}, \hat{\theta}_{n}}^{\prime} \hat{\Lambda}_{n} \dot{f}_{x_{i}, \hat{\theta}_{n}}\right)^{-1}\right) \\
& \times \mathcal{W} i_{d}\left(\Lambda \mid \frac{n+d+1}{2}, \frac{1}{2} \sum_{i=1}^{n}\left(y_{i}-f\left(x_{i}, \hat{\theta}_{n}\right)\right)\left(y_{i}-f\left(x_{i}, \hat{\theta}_{n}\right)\right)^{\prime}\right)
\end{aligned}
$$

which is equal to the density $\hat{p}\left(\theta, \Lambda \mid Z_{n}\right)$ of 4.1 , which is itself consistent by Lemma 4.1. By Proposition 4.4, this density is also a convergent approximation of the posterior density of the parameters.

Let us note that the $\Lambda$ part of this density is Wishart. Due to the assumed Gaussian likelihood of the observations $y_{1}, \ldots, y_{n}$, this density is then a conjugate prior for $\Lambda$ [27]. As a consequence, conditionally on $\theta$, the posterior density of $\Lambda$ is still a Wishart density. This allows the use of a more powerful MCMC algorithm [25, 26]: a hybrid version of the Gibbs and Hastings algorithms. More details are given in the following section. 


\subsection{Hybrid Gibbs-Hastings approximate sampling from $p\left(\theta, \Lambda \mid Z_{n}\right)$}

Let the parameter prior be of the more general form

$$
p(\theta, \Lambda)=h(\theta) \mathcal{W} i_{d}(\Lambda \mid \alpha, \beta)
$$

Then

$$
\begin{aligned}
p\left(\theta, \Lambda \mid Z_{n}\right) & \propto p\left(Z_{n} \mid \theta, \Lambda\right) p(\theta, \Lambda) \\
& \propto \prod_{i=1}^{n} p\left(y_{i}, x_{i} \mid \theta, \Lambda\right) h(\theta) \mathcal{W} i_{d}(\Lambda \mid \alpha, \beta) \\
& \propto \prod_{i=1}^{n} p\left(y_{i} \mid x_{i}, \theta, \Lambda\right) p\left(x_{i} \mid \theta, \Lambda\right) h(\theta) \mathcal{W} i_{d}(\Lambda \mid \alpha, \beta) \\
& \propto \prod_{i=1}^{n} p\left(y_{i} \mid x_{i}, \theta, \Lambda\right) h(\theta) \mathcal{W} i_{d}(\Lambda \mid \alpha, \beta) \\
& \propto|\Lambda|^{n / 2+\alpha-(d+1) / 2} \exp \left\{-\frac{1}{2} \sum_{i=1}^{n}\left\|y_{i}-f\left(x_{i}, \theta\right)\right\|_{\Lambda}^{2}-\operatorname{tr}[\beta \Lambda]\right\} h(\theta)
\end{aligned}
$$

where $h(\theta)$ is supposed to be such that $p\left(\theta, \Lambda \mid Z_{n}\right)$ exists.

(27) shows that conditionally on $\theta$, the marginal $\Lambda$ posterior is then still a Wishart density:

$$
p\left(\Lambda \mid \theta, Z_{n}\right)=\mathcal{W} i_{d}\left(\Lambda \mid \frac{n}{2}+\alpha, \beta+\frac{1}{2} \sum_{i=1}^{n}\left[y_{i}-f\left(x_{i}, \theta\right)\right]\left[y_{i}-f\left(x_{i}, \theta\right)\right]^{\prime}\right)
$$

By contrast, the conditional marginal $\theta$ posterior $p\left(\theta \mid \Lambda, Z_{n}\right)$ is not reachable due to the model nonlinearity with respect to $\theta$ and is only known up to a normalizing constant. Gibbs' algorithm cannot then be used. However, the following hybrid version from [26] or [25] can then be considered

$$
\begin{aligned}
& \text { (i) } \Lambda^{t+1} \sim p\left(\Lambda \mid \theta^{t}, Z_{n}\right) \\
& \text { (ii) } \tilde{\theta} \sim q\left(\theta \mid \theta^{t}, \Lambda^{t+1}\right) \\
& \theta^{t+1}= \begin{cases}\theta^{t} & \text { with probability } 1-\rho \\
\tilde{\theta} & \text { with probability } \rho\end{cases}
\end{aligned}
$$

where $q$ is a proposal distribution for $\theta$, and

$$
\rho=1 \wedge\left\{\frac{p\left(\tilde{\theta} \mid \Lambda^{t+1}, Z_{n}\right)}{q\left(\tilde{\theta} \mid \theta^{t}, \Lambda^{t+1}\right)} / \frac{p\left(\theta^{t} \mid \Lambda^{t+1}, Z_{n}\right)}{q\left(\theta^{t} \mid \theta^{t}, \Lambda^{t+1}\right)}\right\}
$$

in which the ratio $p\left(\tilde{\theta} \mid \Lambda^{t+1}, Z_{n}\right) / p\left(\theta^{t} \mid \Lambda^{t+1}, Z_{n}\right)$ can still be evaluated.

This hybrid algorithm has been shown to combine the advantages of both algorithms of Metropolis-Hastings and Gibbs sampling [25, 26].

Now from a practical point of view we can choose again for $p(\theta, \Lambda)$ the prior given by Equation (25) which satisfies Equation (26), and for the proposal distribution we can choose the restriction to $\theta$ of the distribution $q$ as given by Equation (24):

$$
q\left(\theta^{t+1} \mid \theta^{t}, \Lambda^{t+1}\right)=\mathcal{N}_{s}\left(\theta^{t+1} \mid \theta^{t}, V^{t}\right)
$$

with $V^{t}=\left(\sum_{i=1}^{n} \dot{f}_{x_{i}, \theta^{t}}^{\prime} \Lambda^{t+1} \dot{f}_{x_{i}, \theta^{t}}\right)^{-1}$. 


\subsection{Stopping time of the MCMC generations}

Deciding when to stop the generation of the $\left\{\theta^{k}, \Lambda^{k}\right\}$ Markov chain is an important practical issue. For the following applications, the approach used to monitor the convergence to stationarity is based on comparing parallel simulated sequences. Among the many ways to compare parallel sequences, we employed the approach proposed by [28]. In the applications to follow, 200 trajectories corresponding to different starting points were generated, and stationarity of the chain was assumed when the potential scale reduction $\sqrt{ } \hat{R}$ estimate was below 1.1 , a usual critical value. The quantity $\hat{R}$ is computed from the between and within-sequence variances, see [28] or [26] for more details.

\section{Comparison of criteria performances on case studies}

The analytic posterior predictive density approximation (20) and the two MCMC approximations (Sections 5.1 and 5.2) are used to estimate the expected utility criterion through CV as in Equation (7), on a simulated and a real life model selection problem successively.

These three computing versions of the $U$ criterion are also compared to the standard CV, AIC and BIC criteria on the same case studies:

Two versions of the $\mathrm{CV}$ criterion are considered: the classic one $\mathrm{CV}_{I}=\sum_{i=1}^{n} \| y_{i}-$ $f\left(x_{i}, \hat{\theta}_{n-1}[i]\right) \|_{I_{d \times d}}^{2}$ and a normalized one $\mathrm{CV}_{Q}=\sum_{i=1}^{n}\left\|y_{i}-f\left(x_{i}, \hat{\theta}_{n-1}[i]\right)\right\|_{Q^{-1}}^{2}$ to take account of the correlations between the components of the response vector, where $\hat{\theta}_{n-1}[i]$ are the maximum likelihood estimates of $\theta$ on $Z_{n-1}[i]$ and $Q$ is the empirical variance-covariance matrix of the $\left\{y_{i}\right\}_{i=1, \ldots, n}$. For the AIC and BIC criteria usual forms are considered [2]: AIC = $-2 \log \mathcal{L}\left(\hat{\theta}_{n}, \hat{\Lambda}_{n}\right)+2 K$ and $\mathrm{BIC}=-2 \log \mathcal{L}\left(\hat{\theta}_{n}, \hat{\Lambda}_{n}\right)+K \log n$, where $K$ is the total number of parameters.

We consider first a simulated model selection problem with competing models of low parametric dimension. Secondly, we consider an actual model selection problem from a true dataset. In this second case study, in soil sciences, the competing models are neural networks of large parametric dimension.

\subsection{A simulated model selection problem}

Let us consider the following five two-response regression models:

$$
\begin{gathered}
f_{1}(x, \theta)=\left\{\begin{array}{l}
\theta_{1} \sqrt{x}+\theta_{2} \\
\log x+\theta_{3}
\end{array} \quad f_{2}(x, \theta)=\left\{\begin{array}{l}
\frac{x}{1+\theta_{1} x}+\theta_{2} \\
\log x+\theta_{3}
\end{array} \quad f_{3}(x, \theta)=\left\{\begin{array}{l}
\frac{\theta_{1} x}{\theta_{2}+x}+\theta_{3} \\
\sqrt{x}+\theta_{4}
\end{array}\right.\right.\right. \\
f_{4}(x, \theta)=\left\{\begin{array}{l}
\theta_{1} \log x+\theta_{2} \\
\frac{x}{1+\theta_{3} x}+\theta_{4}
\end{array} \quad f_{5}(x, \theta)=\left\{\begin{array}{l}
\theta_{1} \log x+\theta_{2} \\
\frac{\theta_{3} x}{\theta_{4}+x}+\theta_{5}
\end{array}\right.\right.
\end{gathered}
$$

Data were simulated from model $f_{3}$ with additive centred Gaussian noise of covariance matrix $\Lambda^{-1}=\left[\begin{array}{cc}0.5 & 0.5 \\ 0.5 & 1\end{array}\right]$ and the parameter values $\theta_{1}=2 ., \theta_{2}=1.5, \theta_{3}=1 ., \theta_{4}=1$. Three sets of comparison were carried out with respectively 50,100 and 200 data points for which the $x$ values were sampled from $\mathcal{U}[1,10]$ the uniform distribution on $[1,10]$. Let us note that on this subset of $\mathbb{R}$, the five model functions considered have a comparable behaviour, for a large set of parameter ranges.

The U criterion (7) was first applied according to the three Bayesian CV approaches presented in this paper based on the analytic, the Metropolis-Hastings and the hybrid Gibbs-Hastings posterior 
density approximations respectively. Tables B1, B2 and B3 (Appendix B.1) display the scores reached by the five models (winning scores, which maximize the criterion, are in bold). For the other criteria, AIC, BIC and CV, Tables B4, B5, B6 and B7 (Appendix B.1) display the scores reached by each model (winning scores, which minimize the criterion, are in bold).

Save for the dataset size $n=50$ the analytic and the hybrid Gibbs-Hastings approximations of the $\mathrm{U}$ criterion, always sharply select the true model. The behaviours of AIC and CV are not satisfactory because they end in failure for the largest dataset and offer low contrasted values. That of BIC and that of the Metropolis-Hastings approximation of the U criterion, are completely wrong with no right selection.

The good results of the hybrid Gibbs-Hastings approximation on this example illustrate its superiority over the Metropolis-Hastings one. These two approximations suffer from a rather high variability, the more so as the number of unknown parameters is large. Moreover, a full week of computation with Matlab ${ }^{\circledR}$ on a $3 \mathrm{Ghz}$ Pentium4 was necessary to obtain each of both MCMC approximations of the criterion for each of the five models considered. With these MCMC estimations, the computing time increases dramatically with the number of unknown parameters and the number of observations.

\subsection{An actual model selection problem}

In this second case study, the high parametric dimension of each of the competing models considered (feedforward neural networks) prevented the computation of both posterior predictive density MCMC approximations (Section 5) to estimate the $U$ criterion through CV. Consequently, the computation of the approximation (7) of the $U$ criterion was based on the analytic convergent predictive density approximation (20), for each competing model in each comparison test.

A total of 370 soil layers of Languedoc Plain (south of France) were sampled to study their water retention properties [29]. A soil clod of about $30 \mathrm{~cm}^{3}$ was taken each time. The water contents of the clods at six metric water potentials $(3,10,30,100,300$ and $1500 \mathrm{kPa})$ were measured. Concurrently, nine basic soil variables were also measured for each clod (the bulk density, the proportions of several classes of silt, sand and clay particles and the organic carbon content). A predictive multi-response neural network model with the six water contents as outputs and the nine soil variables as inputs was investigated.

Five quite different feedforward fully connected neural structures, with nine inputs and six outputs, were compared (which were just a few among other possible feedforward neural network structures):

NN1: one hidden layer of 7 neurons (118 parameters).

NN2: two hidden layers of 5 and 4 neurons respectively (104 parameters).

NN3: two hidden layers of 7 and 2 neurons respectively (104 parameters).

NN4: one hidden layer of only 1 neuron (the simplest possible structure, 22 parameters).

NN5: one hidden layer of 10 neurons (166 parameters).

Two types of model comparison were carried out, from the 370 data points:

- In the first one, the $\mathrm{U}, \mathrm{CV}_{Q}$, AIC and BIC criteria were computed for the five neural models using all the 370 data points, each according to its own rule.

- In the second one, the six models were first fitted on a common subset of 320 data points (learning basis) randomly chosen among the 370 of the initial data set. After that, the meansquared error of prediction (MSEP) of each of the five models was computed on the remaining 50 data points (test basis). 
This last procedure was repeated with different randomly sampled 320-point learning bases (and complementary test bases). This MSEP comparison can be considered as a reference for all other types of criterion.

Considering the high number of parameters for each neural model and then the risk of local minima trapping of the least squares parameter estimations, all the necessary fittings were systematically repeated from 50 different starting values randomly chosen in the space of the parameters.

Table B8 (Appendix B.2) shows the score reached by each of the five neural models for each of the four criteria on the full 370-point data set.

Table B9 (Appendix B.2) displays the MSEP values respectively reached by the five models on a typical 50-point test basis.

Both the $\mathrm{U}$ and the $\mathrm{CV}_{Q}$ criteria selected the same neural model, NN1, which coincides with the model of smallest MSEP, whereas both the AIC and BIC criteria wrongly selected the same model, NN5, which presents one of the highest MSEP, but unsurprisingly the lowest number of parameters.

Let us note moreover, that NN1 is not the model with the highest number of parameters, which shows the ability of the $\mathrm{U}$ criterion to trade-off between complexity and over fitting.

All the other model comparisons done with other randomly sampled learning bases and complementary test bases, led to the same relative behaviours of the respective criteria.

\section{Conclusion}

The advantage of predictive distributions in comparing several models in the light of current data has been advocated for long (see [30] for example). Predictive distributions allow diagnostic functions of the local adequacy of the entertained model, to be built from a subsample of the data population and lead to utility functions defined on the whole population [13]. In this framework, this paper shows how a convergent approximation of a predictive distribution of a multiresponse regression model can result from an analytic approach or a numerical MCMC-based one. An approximation of the model expected utility itself is then available which can be computed on a CV-like basis and can be used for ranking competing models. The analytic approximation approach has two main advantages over the numerical approximation ones: on the one hand it is free of any parameter prior density choice and on the other hand it presents very little variability in comparison with that of the numerical MCMC-based approximations. MCMCbased approximations are dependent on the choice of a parameter prior and a proposal distribution, while needing furthermore dramatically heavy computing times even for models of reasonable parametric dimension. It thus seems preferable to use the analytic approach which is in addition much more easier to implement. Finally, comparisons with CV, AIC and BIC criteria on simulated and real data sets of different sizes, on competing models of varied complexity and parameter dimension, show that the performance of the expected utility criterion analytic approximation is much less sensitive to these factors.

\section{References}

[1] G.A.F. Seber and C.F. Wild, Nonlinear Regression, Wiley, NewYork, 1989.

[2] K.P. Burnham and D.R. Anderson, Model Selection and Inference, Springer, NewYork, 1998.

[3] X. Shen and J. Ye, Adaptive model selection, J. Amer. Statist. Assoc. 97 (2002), pp. 210-221.

[4] E.I. George and R. McCullogh, Variable selection via Gibbs sampling, J. Amer. Statist. Assoc. 88 (1993), pp. 881-889.

[5] E.I. George and R. McCullogh, Stochastic search variable selection, in Practical Markov Chain Monte Carlo in Practice, W.R. Gilks, S. Richardson, and D.J. Spiegelhater, eds., Chapman and Hall, London, 1995, pp. $203-214$. 
[6] E.L. George and R. McCullogh, Approaches for Bayesian variable selection, Statist. Sinica 7 (1997), pp. 339-373.

[7] E.I. George and D.P. Foster, The risk inflation criterion for multiple regression, Ann. Statist. 22 (1994), pp. 1947-1975.

[8] J. Rao, Bootstrap choice of cost complexity for better subset selection, Statist. Sinica 9 (1999), pp. $273-288$.

[9] M. Stone, Cross-validatory choice and assessment of statistical predictions, J. R. Statist. Soc. B 36 (1974), pp. 11-147 (with discussion).

[10] J. Shao, Linear model selection by cross-validation, J. Amer. Statist. Assoc. 88 (1993), pp. 486-494.

[11] S. Geisser and W.F. Eddy, A predictive approach to model selection, J. Amer. Statist. Assoc. 74 (1979), pp. $153-160$.

[12] A.E. Gelfand, D.K. Dey, and H. Chang, Model determination using predictive distributions with implementation via sampling-based methods, in Bayesian Statistics 4, J.M. Bernardo, J.O. Berger, A.P. Dawid, and A.F.M. Smith, eds., Oxford University Press, NewYork, 1992, pp. 147-167 (with discussion).

[13] J.M. Bernardo and A.F.M. Smith, Bayesian Theory, Springer, NewYork, 1994.

[14] J.P. Vila, V. Wagner, and P. Neveu, Bayesian nonlinear model selection and neural networks:A conjugate prior approach, IEEE Trans. Neural Netw. 11 (2000), pp. 265-278.

[15] V. Rossi and J.P. Vila, Bayesian multioutput feedforward neural networks comparison: A conjugate prior approach, IEEE Trans. Neural Netw. 17 (2006), pp. 35-47.

[16] H. White, Maximum likelihood estimation of misspecified models, Econometrica 50 (1982), pp. 1-25.

[17] C. Abraham and B. Cadre, Asymptotic properties of posterior distributions derived from misspecified models, Comptes Rendus Académie des Sciences Paris, Series I 335 (2002), pp. 495-498.

[18] A.E. Gelfand and D.K. Dey, Bayesian Model Choice: asymptotics and exact calculations, J. R. Statist. Soc. B 56 (1994), pp. 501-514.

[19] A.E. Gelfand, Model determination using sampling-based methods, in Markov Chain Monte Carlo in Practice, W.R. Gilks, S. Richardson, and D.J. Spiegelhalter, eds., Chapman and Hall, London, 1996, pp. 145-162.

[20] A. Vehtari and J. Lampinen, Bayesian model assessment and comparison using cross-validation predictive densities, Neur. Comput. 14 (2002), pp. 2439-2468.

[21] A. Vehtari and J. Lampinen, Expected utility estimation via cross-validation, in Bayesian Statistics 7, J.M. Bernardo et al., eds., Oxford University Press, 2003, pp. 701-710.

[22] R.H. Berk, Limiting behavior of posterior distribution when the model is incorrect, Ann. Math. Stat. 37 (1966), pp. 51-58.

[23] R.H. Berk, Consistency a posteriori, Ann. Math. Stat. 41 (1970), pp. 894-906.

[24] W.R. Gilks, S. Richardson, and D.J. Spiegelhalter, Markov Chain Monte Carlo in Practice, Chapman and Hall, London, 1996.

[25] L. Tierney, Markov chains for exploring posterior distributions, Ann. Stat. 22 (1994), pp. 1701-1762 (with discussion).

[26] Robert, C. and Casella, G., Monte Carlo Statistical Methods, Springer-Verlag, NewYork, 1999.

[27] A. Gelman, J.B. Carlin, H.S. Stern, and D.B. Rubin, Bayesian Data analysis, Chapman and Hall, London, 1995.

[28] A. Gelman, Inference and monitoring convergence, in W.R. Gilks, S. Richardson, and D.J. Spiegelhalter, eds., Chapman and Hall, London, 1996, pp. 131-143.

[29] D. Leenhardt, M. Voltz, M. Bornand, and R. Webster, Evaluating soil maps for prediction of soil water properties, Eur. J. Soil Sci. 45(3) (1994), pp. 293-301.

[30] G.E.P. Box, Sampling and Bayes inference in scientific modeling, J. R. Statist. Soc. B 26 (1980), pp. 211-252 (with discussion).

[31] G.A.F. Seber, Multivariate Observations, Wiley, New York, 1984.

\section{Appendix A: Proofs}

\section{A.1 Proof of Lemma 4.1}

Let us study first the asymptotic behaviour of $(\theta, \Lambda)$ according to $\hat{p}\left(\theta, \Lambda \mid Z_{n}\right)$.

(i) Expectations: from Equation (10) and assumption $\mathrm{H}_{2}$ we have

$$
\mathrm{E}_{\hat{p}}\left[\theta, \Lambda \mid Z_{n}\right]=\left(\hat{\theta}_{n}, \frac{n+d+1}{n} \hat{\Lambda}_{n}\right) \stackrel{n \rightarrow \infty}{\longrightarrow}\left(\theta_{o}, \Lambda_{o}\right) \text { a.s. }
$$

(ii) Variances:

- Let $\beta_{n}$ be the inverse of the $\theta$ variance. From Equation (10) $\beta_{n}=V(\theta)^{-1}=\sum_{i=1}^{n} \dot{f}_{x_{i}, \hat{\theta}_{n}}^{\prime} \hat{\Lambda}_{n} \dot{f}_{x_{i}, \hat{\theta}_{n}}$.

Let us show that some terms of $\beta_{n}$ grow to infinity with $n$ :

$$
\text { Let } \quad \dot{\beta}_{n}=1 / n \sum_{i=1}^{n} \dot{f}_{x_{i}, \theta_{o}}^{\prime} \Lambda_{o} \dot{f}_{x_{i}, \theta_{o}}, \quad \tilde{\beta}=\mathrm{E}_{x}\left[\dot{f}_{x, \theta_{o}}^{\prime} \Lambda_{o} \dot{f}_{x, \theta_{o}}\right] \quad \text { and } \quad \tilde{\beta}_{n}=1 / n \sum_{i=1}^{n} \dot{f}_{x_{i}, \hat{\theta}_{n}}^{\prime}
$$

$\hat{\Lambda}_{n} \dot{f}_{x_{i}, \hat{\theta}_{n}}$.

As the $\left\{x_{i}\right\}$ are i.i.d. we have by the strong law of large numbers: $\lim _{n \rightarrow \infty} \dot{\beta}_{n}=\tilde{\beta}$.

As $\left(\hat{\theta}_{n}, \hat{\Lambda}_{n}\right)$ converge a.s. to $\left(\theta_{o}, \Lambda_{o}\right)$ on the probability space $(\Omega, \mathcal{U}, P)$, there exists a $P$-negligible subset $D$ of $\mathcal{U}$ such that for all $\omega \in \Omega \backslash D$ one has $\lim _{n \rightarrow \infty}\left(\hat{\theta}_{n}(\omega), \hat{\Lambda}_{n}(\omega)\right)=\left(\theta_{o}, \Lambda_{o}\right)$. Let us consider the event $\omega \in \Omega \backslash D$. 
As the $\left\{x_{i}\right\}$ belong to the compact set $\mathcal{X}$ and $f$ is $C^{1}$, for all $\epsilon>0$ there exists $N_{\epsilon}$ such that for all $n>N_{\epsilon}$ and for all $x \in \mathcal{X}$ one has $\left\|\dot{f}_{x, \hat{\theta}_{n}(\omega)}^{\prime} \hat{\Lambda}_{n}(\omega) \dot{f}_{x, \hat{\theta}_{n}(\omega)}-\dot{f}_{x, \theta_{o}}^{\prime} \Lambda_{o} \dot{f}_{x, \theta_{o}}\right\|<\epsilon$. Then for all $\epsilon>0$ there exists $N_{\epsilon}$ such that for all $n>N_{\epsilon},\left\|\dot{\beta}_{n}-\tilde{\beta}_{n}(\omega)\right\|<\epsilon$. This implies that

$$
\lim _{n \rightarrow \infty} \tilde{\beta}_{n}=\lim _{n \rightarrow \infty} \frac{1}{n} \sum_{i=1}^{n} \dot{f}_{x_{i}, \hat{\theta}_{n}}^{\prime} \hat{\Lambda}_{n} \dot{f}_{x_{i}, \hat{\theta}_{n}}=\tilde{\beta} \text { a.s. }
$$

Then

$$
\lim _{n \rightarrow \infty} \beta_{n}-n \tilde{\beta}=0 \text { a.s. }
$$

and the variance of $\theta$ according to $\hat{p}_{n}$ tends to zero as $n$ tends to infinity.

- Let $\lambda_{i j}$ be the $i j^{\text {th }}$ term of $\Lambda$, which follows the Wishart distribution introduced in Equation (10).

According to [31], $\lambda_{i i} \sim\left((1 / n) \hat{\Lambda}_{n}\right)_{i i} \chi_{(n+d+1) / 2}^{2}$. Then

$$
V\left(\lambda_{i i}\right)=\frac{\left(\hat{\Lambda}_{n}\right)_{i i}^{2}(n+d+1)}{n^{2}} \stackrel{n \rightarrow \infty}{\longrightarrow} 0 \text { a.s. }
$$

Let $l_{i, j}$ be the $d$-vector with 1 at the $i^{t h}$ and $j^{\text {th }}$ components and zero elsewhere. According to [31], $\lambda_{i i}+\lambda_{j j}+2 \lambda_{i j} \sim$ $l_{i, j}\left((1 / n) \hat{\Lambda}_{n}\right) l_{i, j}^{\prime} \chi_{(n+d+1) / 2}^{2}$. Then

$$
V\left(\lambda_{i i}+\lambda_{j j}+2 \lambda_{i j}\right)=\frac{\left(l_{i, j} \hat{\Lambda}_{n} l_{i, j}^{\prime}\right)^{2}(n+d+1)}{n^{2}} \stackrel{n \rightarrow \infty}{\longrightarrow} 0 \text { a.s. }
$$

and then

$$
V\left(\lambda_{i j}\right) \stackrel{n \rightarrow \infty}{\longrightarrow} 0 \text { a.s. }
$$

Now let $A$ be any measurable subset of $\Theta \times \mathcal{S}$ including an open neighbourhood of $\left(\theta_{o}, \Lambda_{o}\right)$. There exists $\epsilon$ such that $B_{\epsilon}\left(\theta_{o}, \Lambda_{o}\right) \subset A$, where $B_{\epsilon}\left(\theta_{o}, \Lambda_{o}\right)$ is the closed parallelotope of side $\epsilon$ centred at $\left(\theta_{o}, \Lambda_{o}\right)$. Moreover, due to the consistency of $\left(\hat{\theta}_{n}, \hat{\Lambda}_{n}\right)$ there exists $N_{\epsilon} \in \mathbb{N}$, such that for all $n>N_{\epsilon}, B_{\epsilon / 2}\left(\hat{\theta}_{n}, \hat{\Lambda}_{n}\right) \subset B_{\epsilon}\left(\theta_{o}, \Lambda_{o}\right)$ a.s., and

$$
\hat{P}\left(B_{\epsilon / 2}\left(\hat{\theta}_{n}, \hat{\Lambda}_{n}\right)\right) \leq \hat{P}\left(B_{\epsilon}\left(\theta_{o}, \Lambda_{o}\right)\right) \leq \hat{P}(A) \text { a.s. }
$$

Let $\eta_{i}, i=1, \ldots, s, s+1, \ldots, s+d(d+1) / 2$, uniformly denote the $s$ components of $\theta$ and the $d(d+1) / 2$ components of $\Lambda$, and $n_{p}=s+d(d+1) / 2$. Let $\hat{\eta}_{i}^{n}=\mathrm{E}_{\hat{p}}\left[\eta_{i}\right]$.

$$
\hat{P}\left(B_{\epsilon / 2}\left(\hat{\theta}_{n}, \hat{\Lambda}_{n}\right)\right)=1-\hat{P}\left(\overline{B_{\epsilon / 2}\left(\hat{\theta}_{n}, \hat{\Lambda}_{n}\right)}\right)=1-\hat{P}\left(\left\{\eta: \max _{i=1, \ldots, n_{p}}\left|\eta_{i}-\hat{\eta}_{i}^{n}\right|>\frac{\epsilon}{2}\right\}\right)
$$

For all $i=1, \ldots, n_{p}$, from Markov inequality: $\hat{P}\left(\left|\eta_{i}-\hat{\eta}_{i}^{n}\right|>\epsilon / 2\right) \leq\left(4 V\left(\eta_{i}\right) / \epsilon^{2}\right)$.

Moreover, for all $i=1, \ldots, n_{p}, \lim _{n \rightarrow \infty} V\left(\eta_{i}\right)=0$, by ii). Then for all $\epsilon>0$ there exists $N_{i} \in \mathbb{N}$ such that $\left(4 V\left(\eta_{i}\right) / \epsilon^{2}\right) \leq \varepsilon$ for $n>N_{i}$

Let $N=\max N_{i}, i=1, \ldots, n_{p}$. Then for all $n>N$ and all $i=1, \ldots, n_{p}$

$$
\hat{P}\left(\left|\eta_{i}-\hat{\eta}_{i}^{n}\right|>\frac{\epsilon}{2}\right) \leq \epsilon \text { and then } \hat{P}\left(\max _{i=1, \ldots, n_{p}}\left|\eta_{i}-\hat{\eta}_{i}^{n}\right|>\frac{\epsilon}{2}\right) \leq \epsilon .
$$

Finally, by (A2), for all $\epsilon>0$ there exists $N \in \mathbb{N}$ such that for $n>N$ it holds

$$
\hat{P}(A) \geq 1-\varepsilon \quad \text { a.s. }
$$

\section{A.2 Proof of Proposition 4.2}

Let us first introduce the following lemma which is necessary to the proof:

Lemma A. 1 Let $f, g$ be functions $\mathbb{R}^{d} \rightarrow \mathbb{R}$. Let $K$ be a connected compact of $\mathbb{R}^{d}$. If $\int_{K} f(x) \mathrm{d} x \neq 0$, if $g$ is continuous, then there exists $x_{0} \in K$ such that

$$
\int_{K} f(x) g(x) \mathrm{d} x=g\left(x_{0}\right) \int_{K} f(x) \mathrm{d} x
$$

Proof Let $a=\left(\int_{K} f(x) g(x) \mathrm{d} x / \int_{K} f(x) \mathrm{d} x\right)$. As $K$ is compact and $g$ is continuous, there exists $m$ and $M$ such that for all $x \in K$, it holds $m \leq g(x) \leq M$. The connexity of $K$ ensures therefore that $g(K)=[m, M]$ and then there exists $x_{o} \in K$ such that $g\left(x_{o}\right)=a$.

Let us note that $C \subset C_{\mathcal{S}}=\operatorname{Proj}_{\Theta}(C) \times \mathcal{S}$. 
Let us show that the Kullback-Leibler distance between $\tilde{p}\left(\theta, \Lambda \mid Z_{n}\right)$ and $p\left(\theta, \Lambda \mid Z_{n}\right)$ over $C$ tends to zero as $n$ tends to infinity. From Equations (9) and (11) we easily have:

$$
\mathcal{K}_{C}(p, \tilde{p})=\int_{C} p\left(\theta, \Lambda \mid Z_{n}\right) \log \frac{p\left(\theta, \Lambda \mid Z_{n}\right)}{\tilde{p}\left(\theta, \Lambda \mid Z_{n}\right)} \mathrm{d} \theta \mathrm{d} \Lambda=\log \frac{K_{n}}{\tilde{K}_{n}} P(C)+E_{p, C}[\log p(\theta, \Lambda)]
$$

where

$$
P(C)=\int_{C} p\left(\theta, \Lambda \mid Z_{n}\right) \mathrm{d} \theta \mathrm{d} \Lambda \quad \text { and }
$$

$E_{p, C}[\log p(\theta, \Lambda)]=\int_{C} \log p(\theta, \Lambda) p\left(\theta, \Lambda \mid Z_{n}\right)$

$\mathrm{d} \theta \mathrm{d} \Lambda$

- Let us study the behaviour of $E_{p, C}[\log p(\theta, \Lambda)]$ :

For all $\varepsilon>0$ let $V_{\varepsilon}=B_{\varepsilon}\left(\theta_{o}, \Lambda_{o}\right)$, the closed ball of radius $\varepsilon$ and centred at $\left(\theta_{o}, \Lambda_{o}\right)$ as defined in Section 2. For $\varepsilon$ sufficiently small it holds $V_{\varepsilon} \subset C$. Therefore,

$$
\begin{aligned}
E_{p, C}[\log p(\theta, \Lambda)]= & \int_{C} \log p(\theta, \Lambda) p\left(\theta, \Lambda \mid Z_{n}\right) \mathrm{d} \theta \mathrm{d} \Lambda \\
= & \int_{C \backslash V_{\varepsilon}} \log p(\theta, \Lambda) p\left(\theta, \Lambda \mid Z_{n}\right) \mathrm{d} \theta \mathrm{d} \Lambda \\
& +\int_{V_{\varepsilon}} \log p(\theta, \Lambda) p\left(\theta, \Lambda \mid Z_{n}\right) \mathrm{d} \theta \mathrm{d} \Lambda
\end{aligned}
$$

Hence

$$
\begin{gathered}
\left|E_{p, C}[\log p(\theta, \Lambda)]-\int_{V_{\varepsilon}} \log p(\theta, \Lambda) p\left(\theta, \Lambda \mid Z_{n}\right) \mathrm{d} \theta \mathrm{d} \Lambda\right| \\
\leq \sup _{\theta, \Lambda \in C}|\log p(\theta, \Lambda)| \int_{C \backslash V_{\varepsilon}} p\left(\theta, \Lambda \mid Z_{n}\right) \mathrm{d} \theta \mathrm{d} \Lambda
\end{gathered}
$$

Let us first note that by $H_{4}$ there exists a finite positive constant $Q(C)$ such that $Q(C)=\sup _{\theta, \Lambda \in C}|\log p(\theta, \Lambda)|$.

We know by $[17,22,23]$ that under $H_{2}, p\left(\theta, \Lambda \mid Z_{n}\right)$ is consistent as $n$ tends to infinity: it concentrates around $\left(\theta_{o}, \Lambda_{o}\right)$, the true parameter values when model $M$ is the correct one, and more generally the parameter values minimizing the Kullback-Leibler criterion between the true $(x, y)$ data distribution and that induced by model $M$ when it is incorrect.

Therefore, for all $\varepsilon>0$ there exists $N_{\varepsilon} \in \mathbb{N}$ such that for all $n \geq N_{\varepsilon}$

$$
\int_{C \backslash V_{\varepsilon}} p\left(\theta, \Lambda \mid Z_{n}\right) \mathrm{d} \theta \mathrm{d} \Lambda<\varepsilon
$$

and

$$
0 \leq P(C)-\int_{V_{\varepsilon}} p\left(\theta, \Lambda \mid Z_{n}\right) \mathrm{d} \theta \mathrm{d} \Lambda<\varepsilon
$$

Moreover by Lemma A1, for all $n$ there exists $(\tilde{\theta}, \tilde{\Lambda}) \in V_{\varepsilon}$ such that

$$
\int_{V_{\varepsilon}} \log p(\theta, \Lambda) p\left(\theta, \Lambda \mid Z_{n}\right) \mathrm{d} \theta \mathrm{d} \Lambda=\log p(\tilde{\theta}, \tilde{\Lambda}) \int_{V_{\varepsilon}} p\left(\theta, \Lambda \mid Z_{n}\right) \mathrm{d} \theta \mathrm{d} \Lambda
$$

By (A6) there exists $\tilde{\varepsilon}: 0 \leq \tilde{\varepsilon} \leq \varepsilon$ such that $\int_{V_{\varepsilon}} p\left(\theta, \Lambda \mid Z_{n}\right) \mathrm{d} \theta \mathrm{d} \Lambda+\tilde{\varepsilon}=P(C)$.

Then

$$
\left|E_{p, C}[\log p(\theta, \Lambda)]-\log p(\tilde{\theta}, \tilde{\Lambda})(P(C)-\tilde{\varepsilon})\right| \leq \varepsilon Q(C)
$$

But $\lim _{\varepsilon \rightarrow 0} \log p(\tilde{\theta}, \tilde{\Lambda})(P(C)-\tilde{\varepsilon})=\log p\left(\theta_{o}, \Lambda_{o}\right) P(C)$. Therefore,

$$
\lim _{n \rightarrow \infty} E_{p, C}[\log p(\theta, \Lambda)]=\log p\left(\theta_{o}, \Lambda_{o}\right) P(C)
$$

- Let us study now the behaviour of $\log \left(K_{n} / \tilde{K}_{n}\right)$ :

For brevity's sake let us denote $f_{n}(\theta, \Lambda)=|\Lambda|^{n / 2} \exp \left\{-1 / 2 \sum_{i=1}^{n}\left\|y_{i}-f\left(x_{i}, \theta\right)\right\|_{\Lambda}^{2}\right\}$.

From Equations (9) and (11): $K_{n}^{-1}=\int f_{n}(\theta, \Lambda) p(\theta, \Lambda) \mathrm{d} \theta \mathrm{d} \Lambda$ and $\tilde{K}_{n}{ }^{-1}=\int_{C_{\mathcal{S}}} f_{n}(\theta, \Lambda) \mathrm{d} \theta \mathrm{d} \Lambda$.

Let $\epsilon>0$ and $V_{\epsilon}=B_{\epsilon}\left(\theta_{o}, \Lambda_{o}\right)$. As $p\left(\theta, \Lambda \mid Z_{n}\right)$ and $\tilde{p}\left(\theta, \Lambda \mid Z_{n}\right)$ are consistent, for all $\epsilon$ there exist $N_{\epsilon}$ and $\tilde{N}_{\epsilon}$ such that for all $n>N_{\epsilon}$

$$
0 \leq 1-K_{n} \int_{V_{\epsilon}} f_{n}(\theta, \Lambda) p(\theta, \Lambda) \mathrm{d} \theta \mathrm{d} \Lambda \leq \epsilon
$$

and for all $n>\tilde{N}_{\epsilon}$

$$
0 \leq 1-\tilde{K}_{n} \int_{V_{\epsilon}} f_{n}(\theta, \Lambda) \mathrm{d} \theta \mathrm{d} \Lambda \leq \epsilon
$$

From Equation (A11) there exists $\tilde{\epsilon} \leq \epsilon$ such that $\int_{V_{\epsilon}} f_{n}(\theta, \Lambda) \mathrm{d} \theta \mathrm{d} \Lambda=(1-\tilde{\epsilon}) / \tilde{K}_{n}$. 
Moreover according to Lemma A.1 there exist $(\tilde{\theta}, \tilde{\Lambda}) \in V_{\epsilon}$ such that

$$
\begin{aligned}
\int_{V_{\epsilon}} f_{n}(\theta, \Lambda) p(\theta, \Lambda) \mathrm{d} \theta \mathrm{d} \Lambda & =p(\tilde{\theta}, \tilde{\Lambda}) \int_{V_{\epsilon}} f_{n}(\theta, \Lambda) \mathrm{d} \theta \mathrm{d} \Lambda \\
& =p(\tilde{\theta}, \tilde{\Lambda}) \frac{1-\tilde{\epsilon}}{\tilde{K}_{n}}
\end{aligned}
$$

which with Equation (A10) gives

$$
\left|1-p(\tilde{\theta}, \tilde{\Lambda}) \frac{K_{n}}{\tilde{K}_{n}}(1-\tilde{\epsilon})\right| \leq \epsilon
$$

By assumption $H_{4} p(\theta, \Lambda)$ is strictly positive and bounded over $V_{\epsilon}=B_{\epsilon}\left(\theta_{o}, \Lambda_{o}\right)$. We deduce that

$$
\lim _{n \rightarrow \infty} \log \frac{K_{n}}{\tilde{K}_{n}}=-\log p\left(\theta_{o}, \Lambda_{o}\right)
$$

By Equations (A9) and (A4) we finally get

$$
\lim _{n \rightarrow \infty} \mathcal{K}_{C}(p, \tilde{p})=0
$$

which leads to Equation (12) and Proposition 4.2, since $L_{1}$-convergence is ensured by Kullback convergence.

\subsection{Proof of Proposition 4.3}

By definition

$$
\tilde{p}\left(\theta, \Lambda \mid Z_{n}\right)=\tilde{K}_{n}|\Lambda|^{n / 2} \exp \left\{-\frac{1}{2} \sum_{i=1}^{n}\left\|y_{i}-f\left(x_{i}, \theta\right)\right\|_{\Lambda}^{2}\right\} \times I_{C_{\mathcal{S}}}(\theta, \Lambda)
$$

and

$$
\hat{p}\left(\theta, \Lambda \mid Z_{n}\right)=\hat{K}_{n}|\Lambda|^{n / 2} \exp \left\{-\frac{1}{2}\left\|\theta-\hat{\theta}_{n}\right\|_{\beta_{n}}^{2}-\frac{1}{2} \sum_{i=1}^{n}\left\|y_{i}-f\left(x_{i}, \hat{\theta}_{n}\right)\right\|_{\Lambda}^{2}\right\}
$$

where $\beta_{n}=\sum_{i=1}^{n} \dot{f}_{x_{i}, \hat{\theta}_{n}}^{\prime} \hat{\Lambda}_{n} \dot{f}_{x_{i}, \hat{\theta}_{n}}$.

Let us denote $E_{\hat{p}, C_{\mathcal{S}}}[]=.\int_{C_{\mathcal{S}}}[.] \hat{p}\left(\theta, \Lambda \mid Z_{n}\right) \mathrm{d} \theta \mathrm{d} \Lambda$.

Let us consider the Kullback-Leibler distance between $\tilde{p}$ and $\hat{p}$ over $C_{\mathcal{S}}$

$$
\begin{aligned}
\mathcal{K}_{C_{\mathcal{S}}}(\hat{p}, \tilde{p})= & \int_{C_{\mathcal{S}}} \hat{p}\left(\theta, \Lambda \mid Z_{n}\right) \log \frac{\hat{p}\left(\theta, \Lambda \mid Z_{n}\right)}{\tilde{p}\left(\theta, \Lambda \mid Z_{n}\right)} \mathrm{d} \theta \mathrm{d} \Lambda \\
= & \log \frac{\hat{K}_{n}}{\tilde{K}_{n}}+E_{\hat{p}, C_{\mathcal{S}}}\left[-\frac{1}{2}\left\|\theta-\hat{\theta}_{n}\right\|_{\beta_{n}}^{2}-\frac{1}{2} \sum_{i=1}^{n}\left\|y_{i}-f\left(x_{i}, \hat{\theta}_{n}\right)\right\|_{\Lambda}^{2}\right] \\
& +E_{\hat{p}, C_{\mathcal{S}}}\left[\frac{1}{2} \sum_{i=1}^{n}\left\|y_{i}-f\left(x_{i}, \theta\right)\right\|_{\Lambda}^{2}\right]
\end{aligned}
$$

Let us consider the successive terms of $\mathcal{K}_{C_{\mathcal{S}}}(\hat{p}, \tilde{p})$ :

- Let $\mathcal{E}_{C_{\mathcal{S}, n}}=E_{\hat{p}, C_{\mathcal{S}}}\left[-(1 / 2)\left\|\theta-\hat{\theta}_{n}\right\|_{\beta_{n}}^{2}\right]$.

By definition of $\hat{p}(\theta, \Lambda)$ in Equation (10): $0>\mathcal{E}_{C_{\mathcal{S}, n}} \geq E_{\hat{p}}\left[-(1 / 2)\left\|\theta-\hat{\theta}_{n}\right\|_{\beta_{n}}^{2}\right]=-s / 2$.

- For all $i=1, \ldots, n$, in a neighbourhood of $\hat{\theta}_{n}$ it holds

$$
\begin{aligned}
\left\|y_{i}-f\left(x_{i}, \theta\right)\right\|_{\Lambda}^{2}= & \left\|y_{i}-f\left(x_{i}, \hat{\theta}_{n}\right)+\dot{f}_{x_{i}, \hat{\theta}_{n}}\left(\theta-\hat{\theta}_{n}\right)\right\|_{\Lambda}^{2}+o\left(\left\|\theta-\hat{\theta}_{n}\right\|^{2}\right) \\
= & \left\|y_{i}-f\left(x_{i}, \hat{\theta}_{n}\right)\right\|_{\Lambda}^{2}+\left\|\dot{f}_{x_{i}, \hat{\theta}_{n}}\left(\theta-\hat{\theta}_{n}\right)\right\|_{\Lambda}^{2} \\
& +2\left\langle y_{i}-f\left(x_{i}, \hat{\theta}_{n}\right), \dot{f}_{x_{i}, \hat{\theta}_{n}}\left(\theta-\hat{\theta}_{n}\right)\right\rangle_{\Lambda}+o\left(\left\|\theta-\hat{\theta}_{n}\right\|^{2}\right)
\end{aligned}
$$

Therefore,

$$
\begin{aligned}
& \frac{1}{2} \sum_{i=1}^{n}\left\|y_{i}-f\left(x_{i}, \theta\right)\right\|_{\Lambda}^{2}-\frac{1}{2} \sum_{i=1}^{n}\left\|y_{i}-f\left(x_{i}, \hat{\theta}_{n}\right)\right\|_{\Lambda}^{2} \\
& \quad=\frac{1}{2} \sum_{i=1}^{n}\left\|\dot{f}_{x_{i}, \hat{\theta}_{n}}\left(\theta-\hat{\theta}_{n}\right)\right\|_{\Lambda}^{2}+\sum_{i=1}^{n}\left\langle y_{i}-f\left(x_{i}, \hat{\theta}_{n}\right), \dot{f}_{x_{i}, \hat{\theta}_{n}}\left(\theta-\hat{\theta}_{n}\right)\right\rangle_{\Lambda}+n o\left(\left\|\theta-\hat{\theta}_{n}\right\|^{2}\right)
\end{aligned}
$$

By Equation (2) and by definition of $\hat{p}\left(\theta, \Lambda \mid Z_{n}\right)$ in Equation (10): $E_{\hat{p}}[\Lambda]=((n+d+1) / n) \hat{\Lambda}_{n}$. 
Therefore,

$$
\begin{aligned}
& E_{\hat{p}, C_{\mathcal{S}}}\left[\sum_{i=1}^{n}\left\|\dot{f}_{x_{i}, \hat{\theta}_{n}}\left(\theta-\hat{\theta}_{n}\right)\right\|_{\Lambda}^{2}\right]=E_{\hat{p}, C_{\mathcal{S}}}\left[E_{\hat{p}}\left[\sum_{i=1}^{n}\left\|\dot{f}_{x_{i}, \hat{\theta}_{n}}\left(\theta-\hat{\theta}_{n}\right)\right\|_{\Lambda}^{2} \mid \theta\right]\right] \\
& =E_{\hat{p}, C_{\mathcal{S}}}\left[\frac{n+d+1}{n} \sum_{i=1}^{n}\left\|\dot{f}_{x_{i}, \hat{\theta}_{n}}\left(\theta-\hat{\theta}_{n}\right)\right\|_{\hat{\Lambda}_{n}}^{2} \mid\right. \\
& =\frac{n+d+1}{n} E_{\hat{p}, C_{\mathcal{S}}}\left[\left\|\theta-\hat{\theta}_{n}\right\|_{\beta_{n}}^{2}\right] \\
& =-2 \frac{n+d+1}{n} \mathcal{E}_{C_{\mathcal{S}}, n}
\end{aligned}
$$

Similarly

$$
\begin{aligned}
E_{\hat{p}, C_{\mathcal{S}}}\left[\sum_{i=1}^{n}\left\langle y_{i}-f\left(x_{i}, \hat{\theta}_{n}\right), \dot{f}_{x_{i}, \hat{\theta}_{n}}\left(\theta-\hat{\theta}_{n}\right)\right\rangle_{\Lambda}\right] \\
=E_{\hat{p}, C_{\mathcal{S}}}\left[E_{\hat{p}}\left[\sum_{i=1}^{n}\left\langle y_{i}-f\left(x_{i}, \hat{\theta}_{n}\right), \dot{f}_{x_{i}, \hat{\theta}_{n}}\left(\theta-\hat{\theta}_{n}\right)\right\rangle_{\Lambda} \mid \theta\right]\right] \\
=\frac{n+d+1}{n} E_{\hat{p}, C_{\mathcal{S}}}\left[\sum_{i=1}^{n}\left\langle y_{i}-f\left(x_{i}, \hat{\theta}_{n}\right), \dot{f}_{x_{i}, \hat{\theta}_{n}}\left(\theta-\hat{\theta}_{n}\right)\right\rangle_{\hat{\Lambda}_{n}}\right] \\
=\frac{n+d+1}{n} E_{\hat{p}, C_{\mathcal{S}}}[0] \\
=0
\end{aligned}
$$

as $\left(\hat{\theta}_{n}, \hat{\Lambda}_{n}\right)$ are least squares estimators.

By Equations (A20) and (A21) the Kullback-Leibler distance between $\tilde{p}$ and $\hat{p}$ comes to

$$
\mathcal{K}_{C_{\mathcal{S}}}(\hat{p}, \tilde{p})=\log \frac{\hat{K}_{n}}{\tilde{K}_{n}}+\mathcal{E}_{C_{\mathcal{S}, n}}-\frac{n+d+1}{n} \mathcal{E}_{C_{\mathcal{S}, n}}+E_{\hat{p}, C_{\mathcal{S}}}\left[n o\left(\left\|\theta-\hat{\theta}_{n}\right\|^{2}\right)\right]
$$

Let us show that $\lim _{n \rightarrow \infty} \mathrm{E}_{\hat{p}, C_{\mathcal{S}}}\left[n o\left(\left\|\theta-\hat{\theta}_{n}\right\|^{2}\right)\right]=0$ :

We saw previously in Section A1 that there exists a matrix $\tilde{\beta}$ such that $\beta_{n}=V(\theta)^{-1} \stackrel{n \rightarrow \infty}{\simeq} n \tilde{\beta}$. As all norms on $\mathbb{R}^{d}$ are equivalent, there exists positive scalars $\alpha_{1}$ and $\alpha_{2}$ such that

$$
\alpha_{1}\left\|\theta-\hat{\theta}_{n}\right\|_{\tilde{\beta}}^{2} \leq\left\|\theta-\hat{\theta}_{n}\right\|^{2} \leq \alpha_{2}\left\|\theta-\hat{\theta}_{n}\right\|_{\tilde{\beta}}^{2}
$$

or

$$
\alpha_{1} n\left\|\theta-\hat{\theta}_{n}\right\|_{\tilde{\beta}}^{2} \leq n\left\|\theta-\hat{\theta}_{n}\right\|^{2} \leq n \alpha_{2}\left\|\theta-\hat{\theta}_{n}\right\|_{\tilde{\beta}}^{2}
$$

and for sufficiently great $n$

$$
\alpha_{1}\left\|\theta-\hat{\theta}_{n}\right\|_{\beta_{n}}^{2} \leq n\left\|\theta-\hat{\theta}_{n}\right\|^{2} \leq \alpha_{2}\left\|\theta-\hat{\theta}_{n}\right\|_{\beta_{n}}^{2}
$$

According to the $\hat{p}$ distribution (10), $\left\|\theta-\hat{\theta}_{n}\right\|_{\beta_{n}}^{2}$ is distributed as a $\chi_{s}^{2}$ variable.

Taking expectations

$$
\alpha_{1} q \leq E_{\hat{p}}\left[n\left\|\theta-\hat{\theta}_{n}\right\|^{2}\right] \leq \alpha_{2} q
$$

There exists then two positive scalars $\tilde{\alpha}_{1}$ and $\tilde{\alpha}_{2}$ such that

$$
\tilde{\alpha}_{1} \leq E_{\hat{p}, C_{\mathcal{S}}}\left[n\left\|\theta-\hat{\theta}_{n}\right\|^{2}\right] \leq \tilde{\alpha}_{2}
$$

Let us come back to the term $o\left(\left\|\theta-\hat{\theta}_{n}\right\|^{2}\right)$ in Equation (A18):

For all couples $\left(x_{i}, y_{i}\right)$ let us denote $g_{n}^{i}(\theta)=o\left(\left\|\theta-\hat{\theta}_{n}\right\|^{2}\right)$. Then

$$
\sum_{i=1}^{n} g_{n}^{i}(\theta)=n o\left(\left\|\theta-\hat{\theta}_{n}\right\|^{2}\right)
$$

Let $\tilde{g}_{n}^{i}(\theta)=\left(g_{n}^{i}(\theta) /\left\|\theta-\hat{\theta}_{n}\right\|^{2}\right)$ and $\tilde{g}_{n}(\theta)=\frac{1}{n} \sum_{i=1}^{n} \tilde{g}_{n}^{i}(\theta)$. Then $\lim _{\theta \rightarrow \hat{\theta}_{n}} \tilde{g}_{n}(\theta)=0$. 
As $\lim _{n \rightarrow \infty} \hat{\theta}_{n}=\theta_{o}$ a.s., for all $i \in \mathbb{N}^{*}$ we have $\lim _{n \rightarrow \infty} \tilde{g}_{n}^{i}\left(\theta_{o}\right)=0$. Moreover as the $\left\{x_{i}\right\}$ belong to the compact subset $\mathcal{X}$ and the model function $f$ is $C^{1}$ with respect to $x$ and $\theta$, the last convergence is uniform with respect to $x$ :

$$
\forall \varepsilon>0, \exists N_{\varepsilon}, \forall x \in \mathcal{X}, \forall n>N_{\varepsilon},\left|\tilde{g}_{n}^{i}\left(\theta_{v}\right)\right|<\varepsilon
$$

and

then

$$
\forall \varepsilon>0, \exists N_{\varepsilon}, \forall n>N_{\varepsilon},\left|\tilde{g}_{n}\left(\theta_{o}\right)\right|<\varepsilon
$$

Now

$$
\lim _{n \rightarrow \infty} \tilde{g}_{n}\left(\theta_{o}\right)=0
$$

$$
E_{\hat{p}, C_{\mathcal{S}}}\left[n o\left(\left\|\theta-\hat{\theta}_{n}\right\|^{2}\right)\right]=E_{\hat{p}, C_{\mathcal{S}}}\left[\tilde{g}_{n}(\theta) n\left\|\theta-\hat{\theta}_{n}\right\|^{2}\right]
$$

For all $\varepsilon>0$, let $V_{\varepsilon}$ be the ball of radius $\varepsilon$ centred at $\theta_{o}, \Lambda_{o}$. Let us choose $\varepsilon$ sufficiently small such that $V_{\varepsilon} \subset C_{\mathcal{S}}$. Then

$$
\begin{aligned}
E_{\hat{p}, C_{\mathcal{S}}}\left[n o\left(\left\|\theta-\hat{\theta}_{n}\right\|^{2}\right)\right]= & \int_{C_{\mathcal{S}} \backslash V_{\varepsilon}} \tilde{g}_{n}(\theta) n\left\|\theta-\hat{\theta}_{n}\right\|^{2} \hat{p}\left(\theta, \Lambda \mid Z_{n}\right) \mathrm{d} \theta \mathrm{d} \Lambda \\
& +\int_{V_{\varepsilon}} \tilde{g}_{n}(\theta) n\left\|\theta-\hat{\theta}_{n}\right\|^{2} \hat{p}\left(\theta, \Lambda \mid Z_{n}\right) \mathrm{d} \theta \mathrm{d} \Lambda
\end{aligned}
$$

As $\hat{p}$ is consistent, for all $\varepsilon>0$ such that $V_{\varepsilon} \subset C_{\mathcal{S}}$ there exists $N_{\varepsilon}$ such that for all $n>N_{\varepsilon}$

$$
\int_{C_{\mathcal{S}} \backslash V_{\varepsilon}} n\left\|\theta-\hat{\theta}_{n}\right\|^{2} \hat{p}\left(\theta, \Lambda \mid Z_{n}\right) \mathrm{d} \theta \mathrm{d} \Lambda<\varepsilon
$$

Then for all $n>N_{\varepsilon}$

$$
\left|E_{\hat{p}, C_{\mathcal{S}}}\left[n o\left(\left\|\theta-\hat{\theta}_{n}\right\|^{2}\right)\right]\right| \leq \sup _{C_{\mathcal{S}}} \tilde{g}_{n}(\theta) \varepsilon+\sup _{V_{\varepsilon}} \tilde{g}_{n}(\theta) \tilde{\alpha}_{2}
$$

As $\sup _{C_{\mathcal{S}}} \tilde{g}_{n}(\theta)$ is bounded, the first term tends to zero with $\varepsilon$. But as $\varepsilon$ tends to zero, $V_{\varepsilon}$ tends to $\left\{\theta_{o}\right\}$. As $n$ tends to $\infty$, then $\sup _{V_{\varepsilon}} \tilde{g}_{n}(\theta)$ tends to $\lim _{n \rightarrow \infty} \tilde{g}_{n}\left(\theta_{o}\right)=0$.

We conclude that

$$
\lim _{n \rightarrow \infty} E_{\hat{p}, C_{\mathcal{S}}}\left[n o\left(\left\|\theta-\hat{\theta}_{n}\right\|^{2}\right)\right]=0
$$

- Finally let us show that $\lim _{n \rightarrow \infty} \log \left(\hat{K}_{n} / \tilde{K}_{n}\right)=0$.

To alleviate notations let us denote, from Equations (A15) and (A16):

$$
\tilde{p}=\tilde{K}_{n} \times \tilde{f}_{n} \quad \text { and } \quad \hat{p}=\hat{K}_{n} \times \hat{f}_{n} .
$$

Let us follow a reductio ad absurdum by assuming that $\lim _{n \rightarrow \infty} \hat{K}_{n} / \tilde{K}_{n} \neq 1$.

Because of Equations (A19)-(A21),

$$
E_{\hat{p}, C_{\mathcal{S}}}\left[\log \frac{\hat{f}_{n}}{\tilde{f}_{n}}\right] \longrightarrow 0 \text { and } E_{\hat{p}, C_{\mathcal{S}}}\left[\log \frac{\tilde{f}_{n}}{\hat{f}_{n}}\right] \longrightarrow 0 \quad \text { as } n \longrightarrow \infty
$$

(i) Suppose first that $\lim _{n \rightarrow \infty} \hat{K}_{n} / \tilde{K}_{n}<1$ :

then

$$
\begin{aligned}
& \lim _{n \rightarrow \infty} E_{\hat{p}, C_{\mathcal{S}}\left[\frac{\tilde{f}_{n}}{\hat{f}_{n}}\right]}=\lim _{n \rightarrow \infty} \hat{K}_{n} \int_{C_{\mathcal{S}}} \tilde{f}_{n} \mathrm{~d} \theta \mathrm{d} \Lambda \\
&<\lim _{n \rightarrow \infty} \tilde{K}_{n} \int_{C_{\mathcal{S}}} \tilde{f}_{n} \mathrm{~d} \theta \mathrm{d} \Lambda \\
&=\lim _{n \rightarrow \infty} \int_{C_{\mathcal{S}}} \tilde{K}_{n} \tilde{f}_{n} \mathrm{~d} \theta \mathrm{d} \Lambda=1
\end{aligned}
$$

Due to the convexity of the exponential function and the consistency of $\hat{p}\left(\theta, \Lambda \mid Z_{n}\right)$ there exists $N$ such that for $n>N$, Jensen inequality can be applied to $E_{\hat{p}, C_{\mathcal{S}}}\left[\left(\tilde{f}_{n} / \hat{f}_{n}\right)\right]$ and gives

$$
\exp E_{\hat{p}, C_{\mathcal{S}}}\left[\log \frac{\tilde{f}_{n}}{\hat{f}_{n}}\right] \leq E_{\hat{p}, C_{\mathcal{S}}}\left[\frac{\tilde{f}_{n}}{\hat{f}_{n}}\right]
$$

but by Equation (A26)

$$
\exp E_{\hat{p}, C_{\mathcal{S}}}\left[\log \frac{\tilde{f}_{n}}{\hat{f}_{n}}\right] \stackrel{n \rightarrow \infty}{\longrightarrow} 1
$$

then $\lim _{n \rightarrow \infty} E_{\hat{p}, C_{\mathcal{S}}}\left[\tilde{f}_{n} / \hat{f}_{n}\right] \geq 1$, which contradicts (A27).

(ii) Suppose now that $\lim _{n \rightarrow \infty}\left(\hat{K}_{n} / \tilde{K}_{n}\right)>1$ : 
By a similar reasoning and since $\tilde{p}\left(\theta, \Lambda \mid Z_{n}\right)$ is consistent

$$
\lim _{n \rightarrow \infty} E_{\hat{p}, C_{\mathcal{S}}}\left[\frac{\tilde{f}_{n}}{\hat{f}_{n}}\right]>\lim _{n \rightarrow \infty} \int_{C_{\mathcal{S}}} \tilde{K}_{n} \tilde{f}_{n} \mathrm{~d} \theta \mathrm{d} \Lambda=1
$$

which implies

$$
\lim _{n \rightarrow \infty} \log E_{\hat{p}, C_{\mathcal{S}}}\left[\frac{\hat{f}_{n}}{\tilde{f}_{n}}\right]<0 .
$$

Due to the convexity of the $\log$ function and the possibility to apply the Jensen inequality to $E_{\hat{p}, C_{\mathcal{S}}}\left[\left(\hat{f}_{n} / \tilde{f}_{n}\right)\right]$, for sufficiently great $n$, we have by Equation (A26)

$$
-\log E_{\hat{p}, C_{\mathcal{S}}}\left[\frac{\hat{f}_{n}}{\tilde{f}_{n}}\right] \leq E_{\hat{p}, C_{\mathcal{S}}}\left[\log \frac{\tilde{f}_{n}}{\hat{f}_{n}}\right] \stackrel{n \rightarrow \infty}{\longrightarrow} 0
$$

which contradicts (A28).

From Equation (i) and (ii) we can deduce that $\lim _{n \rightarrow \infty} \log \left(\hat{K}_{n} / \tilde{K}_{n}\right)=0$, and then, from (A22) and (A25) that

$$
\mathcal{K}_{C_{\mathcal{S}}}(\hat{p}, \tilde{p}) \stackrel{n \rightarrow \infty}{\longrightarrow} 0
$$

This completes the proof of Proposition 4.3, since Kullback convergence dominates $L_{1}$ convergence over $C_{\mathcal{S}}$ and then over $C$.

\section{A.4 Proof of Theorem 4.6}

Let

$$
\begin{aligned}
D= & \int\left|\hat{p}\left(y \mid x, Z_{n}\right)-p\left(y \mid x, Z_{n}\right)\right| \mathrm{d} y \\
= & \int\left|\int \hat{p}(y \mid \theta, \Lambda, x) \hat{p}\left(\theta, \Lambda \mid Z_{n}\right) \mathrm{d} \theta \mathrm{d} \Lambda-\int p(y \mid \theta, \Lambda, x) p\left(\theta, \Lambda \mid Z_{n}\right) \mathrm{d} \theta \mathrm{d} \Lambda\right| \mathrm{d} y \\
= & \int \mid \int p(y \mid \theta, \Lambda, x)\left[\hat{p}\left(\theta, \Lambda \mid Z_{n}\right)-p\left(\theta, \Lambda \mid Z_{n}\right)\right] \mathrm{d} \theta \mathrm{d} \Lambda \\
& +\int \hat{p}\left(\theta, \Lambda \mid Z_{n}\right)[\hat{p}(y \mid \theta, \Lambda, x)-p(y \mid \theta, \Lambda, x)] \mathrm{d} \theta \mathrm{d} \Lambda \mid \mathrm{d} y \\
\leq & \int p(y \mid \theta, \Lambda, x)\left|\hat{p}\left(\theta, \Lambda \mid Z_{n}\right)-p\left(\theta, \Lambda \mid Z_{n}\right)\right| \mathrm{d} \theta \mathrm{d} \Lambda \mathrm{d} y \\
& +\int \hat{p}\left(\theta, \Lambda \mid Z_{n}\right)|\hat{p}(y \mid \theta, \Lambda, x)-p(y \mid \theta, \Lambda, x)| \mathrm{d} \theta \mathrm{d} \Lambda \mathrm{d} y
\end{aligned}
$$

By Fubini's theorem

$$
\begin{aligned}
D \leq & \int\left|\hat{p}\left(\theta, \Lambda \mid Z_{n}\right)-p\left(\theta, \Lambda \mid Z_{n}\right)\right| \mathrm{d} \theta \mathrm{d} \Lambda \\
& +\int \hat{p}\left(\theta, \Lambda \mid Z_{n}\right) \int|\hat{p}(y \mid \theta, \Lambda, x)-p(y \mid \theta, \Lambda, x)| \mathrm{d} y \mathrm{~d} \theta \mathrm{d} \Lambda \\
= & T_{1}+T_{2}
\end{aligned}
$$

As $\hat{p}\left(\theta, \Lambda \mid Z_{n}\right)$ is assumed to be a $L_{1}$-convergent approximation of $p\left(\theta, \Lambda \mid Z_{n}\right), T_{1}$ tends to zero as $n$ tends to $\infty$. Let us show that the same is true for $T_{2}$.

Let $h\left(\theta, \hat{\theta}_{n}\right)=\int|\hat{p}(y \mid \theta, \Lambda, x)-p(y \mid \theta, \Lambda, x)| \mathrm{d} y$. Obviously $0 \leq h(\cdot, \cdot) \leq 2$. The mapping $h$ is continuous and $h\left(\hat{\theta}_{n}, \hat{\theta}_{n}\right)=0$ for all $n \in \mathbb{N}^{*}$. As $\lim _{n \rightarrow \infty}\left(\hat{\theta}_{n}, \hat{\Lambda}_{n}\right)=\left(\theta_{o}, \Lambda_{o}\right)$ a.s., we deduce that $\lim _{n \rightarrow \infty} h\left(\theta_{o}, \hat{\theta}_{n}\right)=0$. Moreover, for all $\varepsilon>0$ there exists a neighbourhood of $\left(\theta_{o}, \Lambda_{o}\right), V_{\varepsilon}$, and an integer $N_{1}$ such that for almost all $(\theta, \Lambda) \in V_{\varepsilon}$ and all $n>N_{1}$ we have $h\left(\theta, \hat{\theta}_{n}\right)<\varepsilon / 2$. 
Let us now split $T_{2}$ according to $V_{\varepsilon}$ :

$$
\begin{aligned}
& T_{2}=\int \hat{p}\left(\theta, \Lambda \mid Z_{n}\right) h\left(\theta, \hat{\theta}_{n}\right) \mathrm{d} \theta \mathrm{d} \Lambda \\
& T_{2}=\int_{V_{\varepsilon}}+\int_{V_{\varepsilon}^{c}} \\
& T_{2} \leq \int_{V_{\varepsilon}^{c}} \hat{p}\left(\theta, \Lambda \mid Z_{n}\right) h\left(\theta, \hat{\theta}_{n}\right) \mathrm{d} \theta \mathrm{d} \Lambda+\varepsilon / 2 \\
& T_{2} \leq 2 \int_{V_{\varepsilon}^{c}} \hat{p}\left(\theta, \Lambda \mid Z_{n}\right)+\varepsilon / 2
\end{aligned}
$$

Due to the consistency of $\hat{p}\left(\theta, \Lambda \mid Z_{n}\right)$ as $n \rightarrow \infty$, there exists an integer $N_{2}$ such that for all $n>N_{2}$ we have $\int_{V_{\varepsilon}^{c}} \hat{p}\left(\theta, \Lambda \mid Z_{n}\right)<\varepsilon / 4$ and then $T_{2}<\varepsilon$.

It follows that $D$ tends to zero as $n$ tends to $\infty$.

\section{Appendix B: Case studies result tables}

\section{B.1 Simulated selection problem}

Table B1. U criterion scoring: analytic approximation.

\begin{tabular}{cccccc}
\hline Nb obs & $f_{1}$ & $f_{2}$ & $\boldsymbol{f}_{\mathbf{3}}$ & $f_{4}$ & $f_{5}$ \\
\hline 50 & -1.5823 & -1.4324 & -1.3177 & -1.3614 & $-\mathbf{1 . 1 1 6 5}$ \\
100 & -1.6620 & -1.5919 & $-\mathbf{1 . 4 2 6 5}$ & -1.6600 & -1.4819 \\
200 & -1.7355 & -1.6668 & $-\mathbf{1 . 4 9 9 8}$ & -1.8800 & -1.5703 \\
\hline
\end{tabular}

Table B2. U criterion scoring: Hastings approximation.

\begin{tabular}{cccccc}
\hline Nb obs & $f_{1}$ & $f_{2}$ & $\boldsymbol{f}_{\mathbf{3}}$ & $f_{4}$ & $f_{5}$ \\
\hline 50 & -1.6814 & -1.7920 & -1.5949 & -1.9814 & $-\mathbf{1 . 3 3 1 3}$ \\
100 & -1.7231 & -1.9211 & -1.5654 & -2.0792 & $-\mathbf{1 . 5 4 0 9}$ \\
200 & -1.7681 & -1.7771 & -1.6188 & -2.1698 & $-\mathbf{1 . 5 8 9 7}$ \\
\hline
\end{tabular}

Table B3. U criterion scoring: hybrid G-H approximation.

\begin{tabular}{lccccc}
\hline Nb obs & $f_{1}$ & $f_{2}$ & $\boldsymbol{f}_{\mathbf{3}}$ & $f_{4}$ & $f_{5}$ \\
\hline 50 & -1.7472 & -1.5171 & -1.5274 & -1.7583 & $-\mathbf{1 . 4 8 0 1}$ \\
100 & -1.7949 & -1.6341 & $-\mathbf{1 . 5 7 0 2}$ & -1.9610 & -1.6757 \\
200 & -1.8846 & -1.7844 & $-\mathbf{1 . 6 3 7 7}$ & -2.0694 & -1.8160 \\
\hline
\end{tabular}

Table B4. AIC criterion scoring $\left(\times 10^{-3}\right)$.

\begin{tabular}{cccccc}
\hline Nb obs & $f_{1}$ & $f_{2}$ & $\boldsymbol{f}_{\mathbf{3}}$ & $f_{4}$ & $f_{5}$ \\
\hline 50 & 0.2131 & $\mathbf{0 . 2 0 9 4}$ & 0.2097 & 0.2113 & 0.2107 \\
100 & 0.4332 & 0.4290 & $\mathbf{0 . 4 2 8 9}$ & 0.4315 & 0.4311 \\
200 & 0.8735 & 0.8662 & 0.8639 & 0.8677 & $\mathbf{0 . 8 6 2 8}$ \\
\hline
\end{tabular}


Table B5. BIC criterion scoring $\left(\times 10^{-3}\right)$.

\begin{tabular}{cccccc}
\hline Nb obs & $f_{1}$ & $f_{2}$ & $\boldsymbol{f}_{\mathbf{3}}$ & $f_{4}$ & $f_{5}$ \\
\hline 50 & 0.2306 & $\mathbf{0 . 2 2 6 8}$ & 0.2330 & 0.2346 & 0.2399 \\
100 & 0.4549 & $\mathbf{0 . 4 5 0 7}$ & 0.4577 & 0.4604 & 0.4672 \\
200 & 0.8993 & $\mathbf{0 . 8 9 2 0}$ & 0.8983 & 0.9021 & 0.9058 \\
\hline
\end{tabular}

Table B6. $\mathrm{CV}_{I}$ criterion scoring.

\begin{tabular}{cccccc}
\hline Nb obs & $f_{1}$ & $f_{2}$ & $\boldsymbol{f}_{\mathbf{3}}$ & $f_{4}$ & $f_{5}$ \\
\hline 50 & 1.2309 & 1.2344 & $\mathbf{1 . 2 0 7 2}$ & 1.2334 & 1.2084 \\
100 & 1.4553 & $\mathbf{1 . 4 4 8 1}$ & 1.4486 & 1.4638 & 1.4725 \\
200 & 1.5382 & 1.5351 & 1.5288 & 1.5286 & $\mathbf{1 . 5 2 1 9}$ \\
\hline
\end{tabular}

Table B7. $\mathrm{CV}_{Q}$ criterion scoring.

\begin{tabular}{cccccc}
\hline Nb obs & $f_{1}$ & $f_{2}$ & $\boldsymbol{f}_{\mathbf{3}}$ & $f_{4}$ & $f_{5}$ \\
\hline 50 & 1.7756 & $\mathbf{1 . 6 4 2 2}$ & 1.6797 & 1.7100 & 1.6611 \\
100 & 1.7283 & 1.6847 & $\mathbf{1 . 6 8 2 0}$ & 1.7035 & 1.6916 \\
200 & 1.7478 & 1.7121 & 1.7006 & 1.7222 & $\mathbf{1 . 6 9 0 0}$ \\
\hline
\end{tabular}

\section{B.2 Actual model selection problem in soil science}

Table B8. Scorings of the five neural models according to the four criteria.

\begin{tabular}{lrrrrr}
\hline Neural model & NN1 & NN2 & NN3 & NN4 & NN5 \\
\hline $\mathrm{U}$ & $\mathbf{1 8 . 4 2 0 2}$ & 17.8137 & 16.4986 & 15.0374 & 17.0372 \\
$\mathrm{CV}$ & $\mathbf{1 . 2 2 7 2}$ & 1.3144 & 1.2456 & 2.1509 & 3.1021 \\
$\mathrm{AIC} \times 10^{-3}$ & 2.2468 & 2.3577 & 2.2575 & $\mathbf{2 . 2 3 3 5}$ & 2.5105 \\
$\mathrm{BIC} \times 10^{-3}$ & 2.7086 & 2.7647 & 2.6645 & $\mathbf{2 . 3 1 9 6}$ & 3.1601 \\
\hline
\end{tabular}

Table B9. A typical MSEP scoring of the neural models.

\begin{tabular}{cccccc}
\hline Neural model & NN1 & NN2 & NN3 & NN4 & NN5 \\
\hline MSEP $\times 10^{-2}$ & $\mathbf{0 . 5 2 2 7}$ & 0.5869 & 0.6126 & 0.6967 & 0.8384 \\
\hline
\end{tabular}

